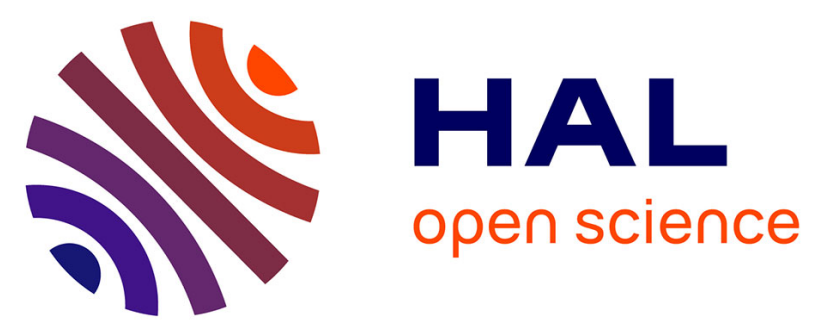

\title{
Random distribution of polydisperse ellipsoidal inclusions and homogenization estimates for porous elastic materials
}

\author{
K. Anoukou, R. Brenner, F. Hong, M. Pellerin, K. Danas
}

\section{To cite this version:}

K. Anoukou, R. Brenner, F. Hong, M. Pellerin, K. Danas. Random distribution of polydisperse ellipsoidal inclusions and homogenization estimates for porous elastic materials. Computers \& Structures, 2018, 10.1016/j.compstruc.2018.08.006 . hal-01875674

HAL Id: hal-01875674

https://hal-polytechnique.archives-ouvertes.fr/hal-01875674

Submitted on 17 Sep 2018

HAL is a multi-disciplinary open access archive for the deposit and dissemination of scientific research documents, whether they are published or not. The documents may come from teaching and research institutions in France or abroad, or from public or private research centers.
L'archive ouverte pluridisciplinaire HAL, est destinée au dépôt et à la diffusion de documents scientifiques de niveau recherche, publiés ou non, émanant des établissements d'enseignement et de recherche français ou étrangers, des laboratoires publics ou privés. 


\title{
Random distribution of polydisperse ellipsoidal inclusions and homogenization estimates for porous elastic materials
}

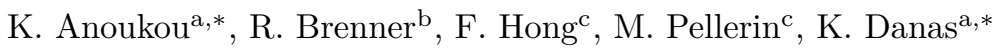 \\ ${ }^{a}$ LMS, CNRS, Ecole Polytechnique, 91128 Palaiseau, France \\ ${ }^{b}$ Sorbonne Université, CNRS, UMR 7190, Institut Jean Le Rond d'Alembert, 75005 Paris, France \\ ${ }^{c}$ Total RED Carbonate-Exploration 8 Production, 64018 Pau, France
}

\begin{abstract}
This work proposes an extension of the well-known random sequential adsorption (RSA) method in the context of non-overlapping random mono- and polydisperse ellipsoidal inclusions. The algorithm is general and can deal with inclusions of different size, shape and orientation with or without periodic geometrical constraints. Specifically, polydisperse inclusions, which can be in terms of different size, shape, orientation or even material properties, allow for larger volume fractions without the need of additional changes in the main algorithm. Unit-cell computations are performed by using either the fast Fourier transformed-based numerical scheme (FFT) or the finite element method (FEM) to estimate the effective elastic properties of voided particulate microstructures. We observe that an isotropic overall response is very difficult to obtain for random distributions of spheroidal inclusions with high aspect ratio. In particular, a substantial increase (or decrease) of the aspect ratio of the voids leads to a markedly anisotropic response of the porous material, which is intrinsic of the RSA construction. The numerical estimates are probed by analytical Hashin-Shtrikman-Willis (HSW) estimates and bounds.
\end{abstract} Key words: RSA algorithm, ellipsoidal inclusions, FFT-based numerical homogenization, effective elastic properties, porous material

\section{Introduction}

The effective physical properties of random heterogeneous materials are strongly dependent upon their microstructure. Therefore, the statistically accurate quantitative characterization of the microstructure is of great importance in their modeling. Specifically, knowledge of microstructural statistical information

5 (e.g. volume fractions, shapes, connectivity, spatial distributions and orientations of constituents) is fundamental in understanding and interpreting the microstructure-property relationships. Statistical descriptors such as $n$-point probability functions, are widely used to quantify mathematically this

\footnotetext{
* Corresponding author.

Email addresses: renald.brenner@sorbonne-universite.fr (R. Brenner), konstantinos.danas@polytechnique.edu (K. Danas)
} 
information in a broad class of random microstructures (see comprehensive reviews in $[1,2]$ ). They are useful in the random generation or stochastic reconstruction of realistic virtual microstructures.

Random generation or stochastic reconstruction of virtual microstructures needs to take into account an infinite amount of microstructural statistical properties. However, in practice, only limited (lower-order) statistical information is available either experimentally or theoretically. The accessibility of the high-level statistical information remains an important focus of research [3]. Statistical microstructural descriptors (e.g., $n$-point probability functions, lineal-path function, pore-size distribution functions, etc.), are widely used to quantify mathematically this information in a broad class of random microstructures. A review of several distribution functions can be found in $[4,2,5]$.

In homogenization based statistical continuum theories $[6,7,8,9,2,10]$, the microstructural statistics of the representative volume element of random multiphase materials is assumed to be spatially uniform and ergodic. This means that probability functions are insensitive to translations and sample realizations. It follows that, the one-point probability function is simply the volume fraction of the phases. In turn, the phase distribution can be described by two- or higher order probability functions and can be statistically isotropic or statistically anisotropic. A number of two-point correlation function methods have been proposed in the literature. The ellipsoidal symmetry can be traced back to Willis [11, 7] while Ponte Castañeda and Willis [12] proposed estimates that allow for different inclusion shapes and distribution functions. In the present work, the focus is on "almost isotropic" responses with randomly oriented ellipsoidal voids and use will be made of the Hashin-Shtrikman-Willis (HSW) estimates $[13,14,15]$, which are considered to be sufficient for the purposes of the present study.

\subsection{Random process based on molecular dynamics}

The basic idea of this process is due to Lubachevsky and Stillinger [16] and Lubachevsky et al. [17] who proposed an algorithm based on molecular dynamics for random packings of disks and spheres in a square primitive cell. This algorithm was extended for a system of non-spherical particles in [18]. The algorithm treats all inclusions on an equal footing consisting in simultaneous generation of inclusions. Recently, Ghossein and Lévesque [19] were inspired by this approach to propose a fully automated numerical tool for a comprehensive validation of linear elastic homogenization models in the case of spherical or ellipsoidal particles reinforced composites. Vincent et al. [20] have used a similar random process based of the molecular dynamics for the study of the effective plastic flow surface of a fluid saturated bi-porous material. The main advantage of this algorithm is that high volume fractions (i.e. greater than $30 \%$ ) can be achieved. Polydisperse microstructures can be simply obtained by considering nonuniform growth rates for the inclusions [21]. 
The algorithm proposed in the present study is based on a consecutive generation of inclusions in a periodic cell. It consists in placing randomly, irreversibly, and sequentially non-overlapping objects into a volume (or onto a surface) $[22,23,2]$. In this process, the acceptance of subsequent inclusion is constrained by some conditions reliant to the previously accepted inclusions and to the cell faces. Recent investigations have used the RSA algorithm in the context of linear and non-linear computational homogenization of composites or porous materials containing spherical particles or pores [24, 25, 26, 27], monodisperse spheroidal inclusions [28] or even inclusions with octahedral, tetrahydral or cubic shapes [29]. The drawback of this algorithm is the fact that in the case of monodisperse microstructure, it is difficult to achieve volume fractions greater than $30 \%$. To overcome this limitation, Segurado and Llorca [24] (see also [30, 31]) developed a modified version of the RSA algorithm applied to spherical particles to reach higher volume fractions. In an alternative perspective and perhaps more closely related to analytical homogenization methods [11, 7], Lopez-Pamies et al. [27] applied the RSA algorithm to polydisperse microstructures allowing for much higher volume fractions without any additional operations and without percolation thresholds (but also look at multiscale morphological models such as in [32] albeit for overlapping inclusions). Most of the studies in the literature pertain to two-phase composites. A recent extension of the RSA algorithms to $N$-phase composites that contain inclusions with different material constitutive response has been proposed in [33].

\subsection{Scope of the study}

In the present study, we propose an extension of the RSA algorithm to obtain random periodic (or not) distributions of mono- and polydisperse non-overlapping ellipsoidal inclusions of arbitrary shape and orientation. The centers of the ellipsoidal inclusions are randomly generated in a cuboidal cell following a uniform distribution probability. All ellipsoids that share the same shape and orientation shall belong to the same particle family. In the case of polydisperse distributions (i.e. inclusions of different sizes), one can reach with appropriate selection of the inter-distance parameters rather high volume fractions $(>60 \%)$ without need of additional operations contrary to previous studies [19]. Then, we consider the numerical computation of the effective linear elastic properties of porous periodic microstructures by using a FFT-based numerical scheme and the FE method. We investigate particularly the deviation from isotropy and compare them with HSW estimates [11] which in some cases correspond to rigorous upper bounds. Voided microstructures are especially of interest in the context of geomaterials and rock physics. We show that the HSW estimates, which correspond to infinitely polydisperse matrix-inclusion systems, are extremely accurate for porous materials (but this is not the case for rigid particles [27]) even for monodisperse microstructures up to fairly high volume fractions (up to 35\%). Beyond that volume fraction, the present algorithm needs to be modified (e.g. 
as in [19]) to reach higher volume fraction of monodisperse inclusions but that is beyond the scope

of this work. Instead, higher and more realistic high-volume-fraction microstructures can be easily attained by using polydisperse sizes of inclusions.

In Section 2, we discuss in detail the extension of the RSA algorithm in the context of random monoand polydisperse ellipsoids. Subsequently, in Section 3, boundary conditions are briefly presented and some details on the FFT and FEM methods, needed to carry out our numerical analysis of the effective elastic properties, are given. In Section 4, we show representative results for "almost" isotropic porous materials with random spherical, oblate and prolate voids and discuss their effect on the effective bulk and shear moduli, as well as their deviation from a purely isotropic response. The complete RSA algorithm is presented in appendix together with a convergence analysis of the FFT computations in terms of number of pores, voxel size and number of realizations.

\section{Extension of the RSA algorithm for ellipsoidal heterogeneities}

In this section, we extend previous approaches to include non-overlapping ellipsoids of arbitrary orientation and relative size. The extension consists in the generalization of the RSA algorithm proposed in $[24,27]$ for polydisperse inclusions. First, we define the microstructural parameters used in our process. Then, similar to most studies in the literature using the RSA algorithms, we introduce the geometric conditions imposed in order to allow for an adequate spacial discretization. Three types of geometric constraints are generally imposed:

1. For non-overlapping inclusions, the spacing between the inclusions must exceed a minimum value noted $s_{1}$ for proper discretization. Assuming periodicity of the inclusion distribution, this condition has to be checked 26 times for each pair of inclusions, i.e. between the newly added inclusion and any previously accepted inclusion as well as its periodic images near the opposite faces.

2. The inclusion surface must be sufficiently far from the cuboidal boundary faces to prevent, for example, the presence of distorted finite elements during meshing. This imposes that the distance from a point on the surface of the inclusion to any of the faces of the cuboidal cell must take a minimum value $s_{2}$. Note, however, that this is less constraining in the context of FFT calculations mainly used in the present work.

3. In the case of periodic microstructures, any inclusion which intersects with any of the cuboidalcell boundary faces must be copied to the opposite face in order to impose periodicity of the microstructure. 
Note that, by contrast with Pierard et al. [34], the extension of the RSA algorithm proposed here takes into account an arbitrary orientation of inclusions. This algorithm can be used to generate random microstructures containing spherical, spheroidal or general ellipsoidal inclusions distributed in a monodisperse or polydisperse manner.

\subsection{Microstructure description}

For simplicity in the following, attention is restricted to inclusions which have the same constitutive properties. The extension to different ones is straightforward and has been described for spherical inclusions in [33].

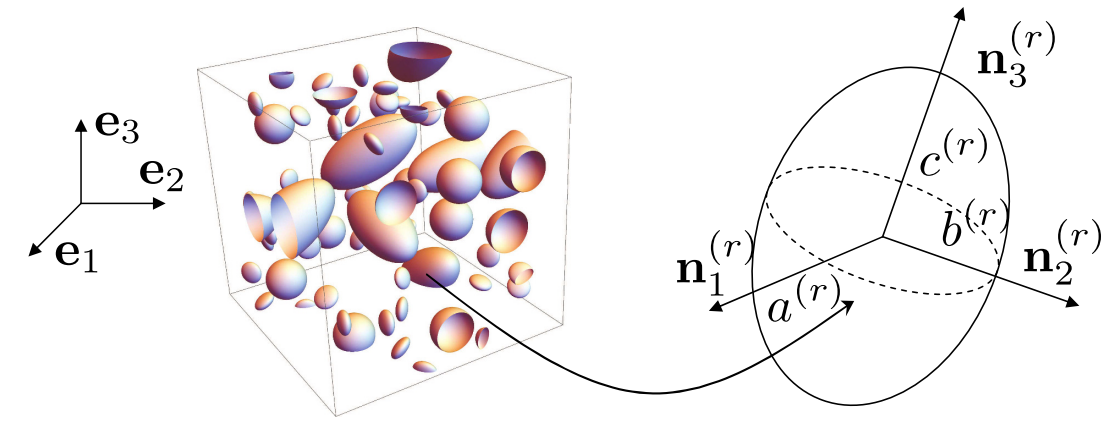

Figure 1: Cubic unit-cell and reference ellipsoid geometry, defined by the aspect ratios $\omega_{1}^{(r)}=c^{(r)} / a^{(r)}$ and $\omega_{2}^{(r)}=c^{(r)} / b^{(r)}$ and the orientation vectors $\mathbf{n}_{i}^{(r)}$ (with $i=1,2,3$ ).

In this study, a monodisperse periodic microstructure is represented by a UC with randomly orientated inclusions having the same size and shape while a polydisperse microsctructure refers to inclusions of different (relative) size and/or shape. Following [27] for the generation of polydisperse microstructure, we define a reference size of inclusion which is used to calibrate every other inclusion phase/size. The reference size, for consistency with the monodisperse microstructures, is defined via a reference number of monodisperse inclusions, $N^{r e f}$, so that $N^{r e f} \leq N$ with $N=\sum_{r=2}^{N_{p}} N^{(r)}$ being the total number of inclusions with volume fraction $f=\sum_{r=2}^{N_{p}} f^{(r)}$ and aspect ratios $\omega_{1}^{r e f}$ and $\omega_{2}^{r e f}$. The number $N^{r e f}$, the volume fraction $f$ and the reference shape $\left(\omega_{1}^{r e f}, \omega_{2}^{r e f}\right)$ allows to obtain uniquely the reference size of the inclusions in the unit-cell. In the following, we identify the different cases resulting from the above descriptions. 


\subsubsection{Polydisperse only in size}

We consider inclusions of different size but same shape implying that the shape of all inclusions coincides with the reference one: $\omega_{1}^{r e f}=\omega_{1}^{(r)}$ and $\omega_{2}^{r e f}=\omega_{2}^{(r)}\left(r=2, \cdots, N_{p}\right)$. For instance, one can take the first family of inclusions to be the reference one and set one of its axes, say for instance $c^{r e f}$ equal to 1 . The lengths of the semi-axes of the reference inclusions are then given by

$$
a^{r e f}=\frac{c^{r e f}}{\omega_{1}^{r e f}}, \quad b^{r e f}=\frac{c^{r e f}}{\omega_{2}^{r e f}}, \quad c^{r e f}=\left(\frac{3 V f \omega_{1}^{r e f} \omega_{2}^{r e f}}{4 \pi N^{r e f}}\right)^{1 / 3} .
$$

The semi-axes lengths of the other inclusions are determined by using $c^{r e f}$ and the size coefficient $\chi^{(r)}$, such that

$$
c^{(r)}=\chi^{(r)} c^{r e f} \quad a^{(r)}=\frac{c^{(r)}}{\omega_{1}^{(r)}}, \quad b^{(r)}=\frac{c^{(r)}}{\omega_{2}^{(r)}}, \quad \chi^{(r)} \leq 1 .
$$

\subsubsection{Polydisperse only in shape}

In this case, the inclusions are defined by different shapes (i.e. different $\omega_{1}^{(r)}$ and $\omega_{2}^{(r)}$ for different $r$ ) but have the same volume. The reference inclusion is then for simplicity taken to be a sphere of radius $R^{r e f}$, and the calibration is done with respect to the volume of this sphere by setting

$$
R^{r e f}=\left(\frac{3 V f^{r e f}}{4 \pi N^{r e f}}\right)^{1 / 3}
$$

This amounts to define the characteristic lengths of the semi-axes of each family of inclusion as

$$
a^{(r)}=\frac{c^{(r)}}{\omega_{1}^{(r)}}, \quad b^{(r)}=\frac{c^{(r)}}{\omega_{2}^{(r)}}, \quad c^{(r)}=\left(\omega_{1}^{(r)} \omega_{2}^{(r)}\right)^{1 / 3} R^{r e f} .
$$

In this case, there is no preferential order in the random generation of inclusion/phases since they all have the same relative size. One can then begin with the phase $r$ which has the smallest volume fraction $f^{(r)}$ and progress until $\sum_{r=2}^{N_{p}} f^{(r)} \simeq f$. It is worth noticing that instead of using a reference number of inclusions $N^{r e f}$ to define a reference size, one can use directly a reference radius $R^{r e f}$. 


\subsubsection{Polydisperse in shape and size}

This case is a simple combination of the two previous cases. In this general case, the relative size of each family of inclusions is determined using $V^{(r)}=4 \pi\left(\chi^{(r)} R^{r e f}\right)^{3} / 3$, where $R^{r e f}$ is given by (3). Again, the sequential addition process needs not to have a preferential order neither in terms of size nor in shape. Nonetheless, as mentioned previously, starting with the largest size of inclusion proves useful for attaining a better accuracy on the volume fraction as well as for reaching higher compacities.

\subsection{Description of the extended RSA algorithm}

In this section, we describe briefly the main steps of the extended RSA algorithm (see A for more details). The inputs of the algorithm are: the dimensions of the cuboidal cell $L_{1}, L_{2}$ and $L_{3}$, the volume fraction $f$, the number of phases $N_{p}$, the number $N^{r e f}$ of reference inclusions, the microstructural parameters $\omega_{1}^{(r)}, \omega_{2}^{(r)}, \chi^{(r)}, f^{r}$ and two offsets distance $\xi_{1}$ and $\xi_{2}$ used in the calculation of the minimum distance parameters $s_{1}$ and $s_{2}$, respectively. In this work, these parameters are fixed at $\xi_{1}=0.02$ and $\xi_{2}=0.05$, however they are user-defined and can be changed at will. In general, the highest the volume fraction the smallest should be $\xi_{1}$ and $\xi_{2}$. When the desired volume fraction of the inclusions is moderate to low $(<20 \%)$, one can increase those values to obtain more uniform distributions. The outputs of the algorithm are: the position vector of the center of the ellipsoid $\mathbf{v}_{i}^{(r)}$, the semi-axes lengths $\left(a_{i}^{(r)}, b_{i}^{(r)}, c_{i}^{(r)}\right.$ and the Euler angles $\left(\phi_{i}^{(r)}, \theta_{i}^{(r)}, \psi_{i}^{(r)}\right)$ of the ellipsoidal inclusion $i$ belonging to the phase $r$. To determine periodic images of an inclusion, 26 vectors $\mathbf{m}=\left(m_{1}, m_{2}, m_{3}\right)$ are defined, where $m_{1}, m_{2}$ and $m_{3}$ take the values $\left(0,-L_{1}, L_{1}\right),\left(0,-L_{2}, L_{2}\right)$ and $\left(0,-L_{3}, L_{3}\right)$, respectively.

Specifically, for each phase $r=2, \cdots, N_{p}$, the RSA algorithm can be decomposed into four steps:

Step 1: Compute the semi-axis lengths of inclusion $(i)$

$$
c_{i}^{(r)}=\chi^{(r)}\left(\omega_{1}^{(r)} \omega_{2}^{(r)}\right)^{1 / 3} R^{r e f}, \quad b_{i}^{(r)}=c_{i}^{(r)} / \omega_{2}^{(r)} \quad \text { and } \quad a_{i}^{(r)}=c_{i}^{(r)} / \omega_{1}^{(r)}
$$

with $R^{r e f}$ given by (1). For non-spherical inclusions, specify the orientation with Euler angles $\left(\phi_{i}^{(r)}, \theta_{i}^{(r)}, \psi_{i}^{(r)}\right)$.

Step 2: In the sequential addition, generate a random center position vector $\mathbf{v}_{i}^{(r)}$ for inclusion $i$ in phase $r$. Compute the minimum distance $\Delta_{1}$ between a new inclusion $i$ and any previously accepted inclusion $j=1, \ldots, i-1$ including its 26 periodic images, and compare this distance to the minimum value $s_{1}$

$$
s_{1}=\left(\max \left(a_{i}^{(r)}, b_{i}^{(r)}, c_{i}^{(r)}\right)+\max \left(a_{j}^{(r)}, b_{j}^{(r)}, c_{j}^{(r)}\right)\right) * \xi_{1}
$$

In this step, the algorithm for finding the minimum distance between two ellipsoids (see Algorithm 
2 in A) is called. If $\Delta_{1}<s_{1}$, generate a new inclusion center position and recheck the overlapping and minimum distance from existing ellipsoids. If $\Delta_{1} \geq s_{1}$ proceed to Step 3.

Step 3: Use the algorithm for finding the minimum distance between ellipsoid and plane (see Algorithm 3 in A) to determine $\Delta_{2}$ and $\bar{\Delta}_{2}$. Compare the distance $\Delta_{2}$ with the minimum value $s_{2}$

$$
s_{2}=\left(\max \left(a_{i}^{(r)}, b_{i}^{(r)}, c_{i}^{(r)}\right)\right) * \xi_{2}
$$

If $\Delta_{2}<s_{2}$, then generate a new center position of inclusion $i$, otherwise accept the inclusion $i$.

Step 4: Ensure periodicity of the cuboidal cell by considering periodic images of the inclusion (see Section 2.5 below).

In the following, we describe in details the last three steps of the RSA procedure.

\subsection{RSA Step 2: Minimum distance between two ellipsoids}

In this section, we discuss the evaluation of the distance between two ellipsoidal inclusions. This step is extremely important since it allows to decide if a newly added inclusion is accepted or rejected. Specifically, the distance between two non-spherical inclusions is not simply determined by using the center-to-center distance, as is the case with spheres. There are many studies in literature devoted to the determination of the minimum distance between two implicit algebraic surfaces. For example, in the recent work of Chen et al. [35], a useful analytical method has been proposed to compute this distance. The drawback is that this method leads to a very complicated system of three non-linear equations of degrees 2, 3 and 6 to be solved, at least in the case of two ellipsoids. In the work of Pierard et al. [34] on elasto-plastic composite materials reinforced with aligned elastic ellipsoidal particles, an algorithm proposed by Lin and Han [36] is used to determine iteratively the minimum distance between two ellipsoids. This algorithm based on the local approximation of the ellipsoid by a spherical ball has excellent convergence properties. This approach is simple and easy to implement in the present study. Hence, following [36], we implement and embed in the RSA algorithm a slightly modified version of the algorithm. The modification consists in a much simpler and more general way to check the non-overlapping condition of inclusions during the sequential addition. In Lin's algorithm, the non-overlapping is checked by solving two one-dimensional quadratic inequalities at each iteration. With our modification, we are able to check this condition once by using a lemma on the intersection of ellipsoids proposed in the book of Kurzhanski and Vályi [37]. For clarity, in this section, the superscript $(r)$ denoting the different ellipsoidal families is omitted. 


\subsubsection{Statement of the problem}

Given two non-overlapping arbitrarily oriented ellipsoids $\mathcal{E}_{1}$ and $\mathcal{E}_{2}$ defined respectively by $\mathcal{E}_{n}=$ $\mathcal{E}\left(\mathbf{v}_{n} ; \mathbb{Z}_{n}\right), n=1,2$, such that

$$
\mathcal{E}\left(\mathbf{v}_{n} ; \mathbb{Z}_{n}\right):=\left\{\mathbf{x}: Q_{n}(\mathbf{x}) \leq 0\right\}
$$

$Q_{n}$ denotes a quadratic function which reads

$$
Q_{n}(\mathbf{x})=\left(\mathbf{x}-\mathbf{v}_{n}\right)^{\mathrm{T}} \mathbb{Z}_{n}\left(\mathbf{x}-\mathbf{v}_{n}\right)-1
$$

with $\mathbf{v}_{n}$ rhe vector position of the center of the ellipsoid. In turn, the matrix $\mathbb{Z}_{n}$ describess the shape and the orientation of the ellipsoid $\mathcal{E}_{n}$ and is a positive definite square $3 \times 3$ matrix. Its eigenvalues are the reciprocals of the squares of the semi-axes lengths of the ellipsoid (i.e., $a, b, c$ and equivalently the aspect ratios $\omega_{1}$ and $\left.\omega_{2}\right)$ and its eigenvectors $\mathbf{n}_{i}(i=1,2,3)$ define the principal axes of the ellipsoid $\mathcal{E}_{n}$ (Figure 1). For an arbitrarily oriented ellipsoid $\mathcal{E}(\mathbf{v} ; \mathbb{Z})$ with center position $\mathbf{v}$, the matrix $\mathbb{Z}$ is defined as

$$
\mathbb{Z}=\frac{1}{c^{2}}\left(\omega_{1}^{2} \mathbf{n}_{1} \otimes \mathbf{n}_{1}+\omega_{2}^{2} \mathbf{n}_{2} \otimes \mathbf{n}_{2}+\mathbf{n}_{3} \otimes \mathbf{n}_{3}\right)
$$

The orientation of the ellipsoid is specified by the rotation matrix $\mathbb{R}(\phi, \theta, \psi)$ such that $\mathbf{n}_{i}=\mathbb{R} \mathbf{e}_{i}$ $(i=1,2,3)$. $\left(\mathbf{e}_{1}, \mathbf{e}_{2}, \mathbf{e}_{3}\right)$ defines an orthonormal basis of the reference frame (Figure 1). When $\mathbb{R}=\mathbb{I}, \mathcal{E}$ is thus an axis-aligned ellipsoid (i.e. its principal axes coincide with the reference frame axes). The minimum distance between two ellipsoids reads

$$
d\left(\mathcal{E}_{1}, \mathcal{E}_{2}\right)=\min _{\mathbf{x}_{1} \in \Omega_{1}, \mathbf{x}_{2} \in \Omega_{2}}\left\|\mathbf{x}_{1}-\mathbf{x}_{2}\right\|
$$

190

\subsubsection{Algorithm on the local approximation of the ellipsoid by ball}

Before we proceed further, it is recalled that one of the main conditions that need to be checked is that the distance between the ellipsoids shall not exceed a user-defined value $s_{1}$. Therefore, the procedure of evaluating the distance between two different ellipsoids can be split in two main parts. The first part consists in checking whether the ellipsoids overlap/intersect. Provided that the ellipsoids do not overlap, one can then proceed to the second part and evaluate the distance between the two ellipsoids.

First part. In the present work, we propose a simple method to check if the ellipsoids intersect at 
one or more point by using the lemma of Kurzhanski and Vályi [37] which reads: ${ }^{1}$

Two ellipsoids $\mathcal{E}_{1}$ and $\mathcal{E}_{2}$ have a nonempty intersection if, and only if, there exists an ellipsoid $\mathcal{E}_{\alpha}=\mathcal{E}\left(\mathbf{v}_{\alpha} ; \mathbb{Z}_{\alpha}\right)$, for any $\alpha \in[0,1]$, such that $\operatorname{det}\left(\mathbb{Z}_{\alpha}\right)>0$ with

$$
\mathbb{Z}_{\alpha}=\frac{1}{1-h_{\alpha}} \sum_{n=1}^{2} \alpha_{n} \mathbb{Z}_{n}, \quad \alpha_{1}=\alpha, \quad \alpha_{2}=1-\alpha
$$

where

$$
h_{\alpha}=\sum_{n=1}^{2} \alpha_{n}\left(\mathbf{v}_{n} \cdot \mathbb{Z}_{n} \cdot \mathbf{v}_{n}\right)-\left(\sum_{n=1}^{2} \alpha_{n} \mathbb{Z}_{n} \cdot \mathbf{v}_{n}\right) \cdot \mathbf{v}_{\alpha} \quad \text { and } \quad \mathbf{v}_{\alpha}=\left(\sum_{n=1}^{2} \alpha_{n} \mathbb{Z}_{n}\right)^{-1}\left(\sum_{n=1}^{2} \alpha_{n} \mathbb{Z}_{n} \cdot \mathbf{v}_{n}\right)
$$
of ellipsoids.

Second part. If the ellipsoids do not intersect, we proceed to solve the minimization problem (8). The basic idea for solving numerically this problem is due to Lin and Han [36], who proposed an iterative resolution in which each ellipsoid $\mathcal{E}_{n}$ is approximated locally in its interior by a spherical ball which marches along the internal surface of the ellipsoid, i.e. the spherical ball is tangent to the ellipsoid at a single point $\mathbf{x}_{n} \in \Omega_{n}$ (Figure 2$)$. The spherical ball $\mathcal{B}_{n}=\mathcal{B}\left(\mathbf{c}_{n} ; r_{n}\right)(n=1,2)$ is defined by

$$
\mathcal{B}\left(\mathbf{c}_{n} ; r_{n}\right):=\left\{\mathbf{y}:\left\|\mathbf{y}-\mathbf{c}_{n}\right\| \leq r_{n}\right\}
$$

with

$$
\mathbf{c}_{n}=\mathbf{x}_{n}-\frac{\gamma_{n}}{2} \mathbf{N}_{n} \quad \text { and } \quad r_{n}=\frac{\gamma_{n}}{2}\left\|\mathbf{N}_{n}\right\|
$$

Here, $\mathbf{N}_{n}=\nabla Q_{n}$ is the normal to the ellipsoid $\mathcal{E}_{n}$ at the point $\mathbf{x}_{n} \in \Omega_{n}$. The parameter $\gamma_{n}$ is a measure (matrix norm) of the matrix $\mathbb{Z}_{n}$ related to its spectral radius $\rho\left(\mathbb{Z}_{n}\right)$, such that

$$
0<\gamma_{n} \leq \frac{1}{\rho\left(\mathbb{Z}_{n}\right)} \quad \text { and } \quad \gamma_{n}=\frac{1}{\left\|\mathbb{Z}_{n}\right\|_{F}}
$$

where $\|\bullet\|_{F}$ indicates the Frobenius (Euclidean) norm.

The algorithmic process involving both parts is schematically explained in Figure 2 and can be described as follows:

Step 1: Check the overlapping at the beginning of iterations using $(9)$. If $\operatorname{det}\left(\mathbb{Z}_{\alpha}\right)>0$, then set the minimum distance $d\left(\mathcal{E}_{1}, \mathcal{E}_{2}\right)=0$ and terminate, otherwise continue to Initialization.

\footnotetext{
${ }^{1}$ Alternatively, one could check the intersection of two ellipsoids by solving two one-dimensional quadratic inequalities at each iteration, as in [36], but this is more time consuming.
} 


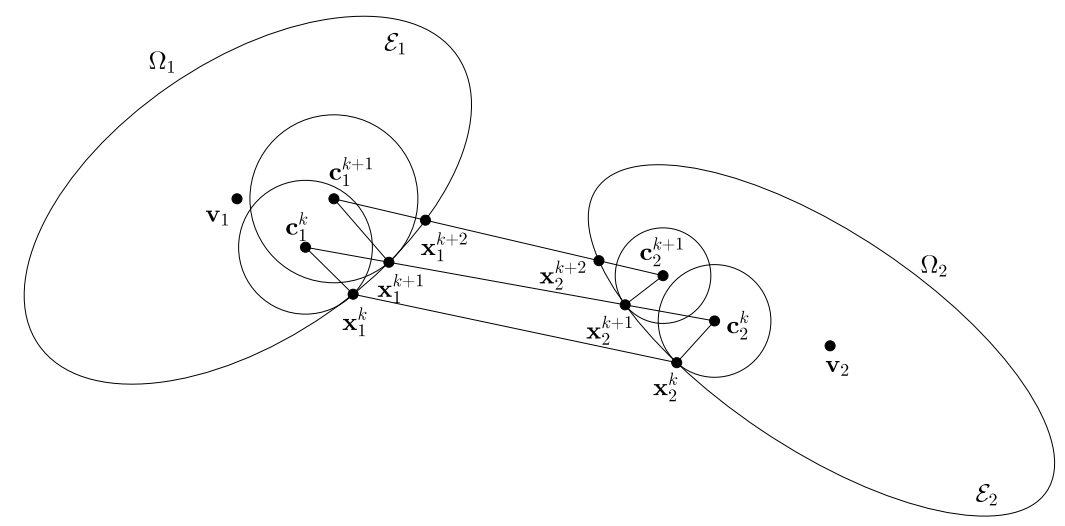

Figure 2: Geometrical representation of iterative steps for finding the minimum distance between two ellipsoids.

Initialization: Choose the centers $\mathbf{v}_{1}$ and $\mathbf{v}_{2}$ of $\mathcal{E}_{1}$ and $\mathcal{E}_{2}$ as starting interior points $\mathbf{c}_{1}$ and $\mathbf{c}_{2}$, respectively.

Step 2: Let us denote by $\mathbf{c}_{1}^{k}$ and $\mathbf{c}_{2}^{k}$ two arbitrary interior points at the $k^{\text {th }}$ iteration of the ellipsoids $\mathcal{E}_{1}$ and $\mathcal{E}_{2}$, respectively. We generate two points $\mathbf{x}_{1}^{k+1}$ and $\mathbf{x}_{2}^{k+1}$ as the intersections of the segment $\left[\mathbf{c}_{1}^{k}, \mathbf{c}_{2}^{k}\right]$ with the ellipsoid surfaces $\Omega_{1}$ and $\Omega_{2}$, so that $\mathbf{x}_{1}^{k+1}=\mathbf{c}_{1}^{k}+t_{1}\left(\mathbf{c}_{2}^{k}-\mathbf{c}_{1}^{k}\right)$ and $\mathbf{x}_{2}^{k+1}=\mathbf{c}_{1}^{k}+t_{2}\left(\mathbf{c}_{2}^{k}-\mathbf{c}_{1}^{k}\right)$, where $t_{1}$ and $t_{2}$ are solutions of the two one-dimensional quadratic equations:

$$
t_{n}=\left\{t \in[0,1]: A_{n} t^{2}+B_{n} t+C_{n}=0\right\}, \quad n=1 \text { or } 2,
$$

with $A_{n}=\left(\mathbf{c}_{2}^{k}-\mathbf{c}_{1}^{k}\right)^{\mathrm{T}} \mathbb{Z}_{n}\left(\mathbf{c}_{2}^{k}-\mathbf{c}_{1}^{k}\right), B_{n}=\left(\mathbf{c}_{1}^{k}-\mathbf{v}_{n}\right)^{\mathrm{T}} \mathbb{Z}_{n}\left(\mathbf{c}_{2}^{k}-\mathbf{c}_{1}\right)$ and $C_{n}=\left(\mathbf{c}_{1}^{k}-\mathbf{v}_{n}\right)^{\mathrm{T}} \mathbb{Z}_{n}\left(\mathbf{c}_{1}^{k}-\mathbf{v}_{n}\right)$. These two points are the closest points and hence the best candidate of the minimum distance if and only if the normals $\mathbf{N}_{1}^{k+1}$ and $\mathbf{N}_{2}^{k+1}$ and the vector $\mathbf{x}_{1}^{k+1}-\mathbf{x}_{2}^{k+1}$ are colinear.

Step 3: If the angles $\angle\left(\mathbf{N}_{1}^{k+1}, \mathbf{x}_{2}^{k+1}-\mathbf{x}_{1}^{k+1}\right)$ and $\angle\left(\mathbf{N}_{2}^{k+1}, \mathbf{x}_{1}^{k+1}-\mathbf{x}_{2}^{k+1}\right)$ are null, set $d\left(\mathcal{E}_{1}, \mathcal{E}_{2}\right)=$ $\left\|\mathbf{x}_{1}^{k+1}-\mathbf{x}_{2}^{k+1}\right\|$, accept the new ellipsoid and move on to the addition of a new ellipsoid, otherwise continue to Step 4 .

Step 4: Construct two new ball centers $c_{1}^{k+1}$ and $c_{2}^{k+1}$ of $\mathcal{B}_{1}$ and $\mathcal{B}_{2}$, respectively, as

$$
\mathbf{c}_{n}^{k+1}=\mathbf{x}_{n}^{k+1}-\frac{\gamma_{n}}{2} \mathbf{N}_{n}^{k+1}, \quad n=1,2
$$

and return to Step 2.

This algorithm is given in full detail in A. 


\subsection{RSA Step 3: Minimum distance between an ellipsoid and a plane}

In this section, we determine the minimum distance between an ellipsoid $\mathcal{E}$ and a plane $\mathcal{P}$. It is worth noting that if the ellipsoid intersects a plane, the terminology minimum distance from a point lying on an ellipsoid to a plane is more appropriate. Then, this distance is obtained by solving a geometrical problem for finding the closest point on the boundary $\Omega$ of the ellipsoid $\mathcal{E}$ where the normal $\mathbf{N}=\nabla Q(\mathbf{x})$ is collinear to the normal of the plane. Knowing this closest point, one can verify the second geometric condition and then impose the periodicity condition mentioned previously.

Any surface of the cuboidal cell is defined by a plane equation given as

$$
\mathcal{P}(\mathbf{n}, \eta):=\{\mathbf{y}: \mathbf{y} \cdot \mathbf{n}+\eta=0\}
$$

where $\mathbf{n}$ is the normal unit vector of the plane $\mathcal{P}$, and $d$ is a constant obtained as a scalar product of $\mathbf{n}$ and a known point on $\mathcal{P}$. For a cuboidal cell with dimensions $L_{1}, L_{2}$ and $L_{3}$, with a corner as origin, let us adopt the following convention: $\mathbf{n}=(-1,0,0)$ and $\eta=0 \rightarrow$ face $1, \mathbf{n}=(1,0,0)$ and $\eta=-L_{1}$ $\rightarrow$ face $2, \mathbf{n}=(0,-1,0)$ and $\eta=0 \rightarrow$ face $3, \mathbf{n}=(0,1,0)$ and $\eta=-L_{2} \rightarrow$ face $4, \mathbf{n}=(0,0,-1)$ and $\eta=0 \rightarrow$ face 5 and $\mathbf{n}=(0,0,1)$ and $\eta=-L_{3} \rightarrow$ face 6 . The problem to solve then reads

$$
\mathbf{N} \times \mathbf{n}=\mathbf{0} \quad \text { and } \quad Q(\mathbf{x})=0 .
$$

The solutions of (15) are two points $\mathbf{x}_{1}$ and $\mathbf{x}_{2}$ representing the closest and the farthest points, or vice versa depending on the face of the cuboidal cell. The related minimum or the maximum distance, denoted as $d(\mathcal{E}, \mathcal{P})$, is obtained by using the formula giving the distance of a point $\mathbf{x}$ to a plane

$$
d(\mathcal{E}, \mathcal{P})=\frac{|\mathbf{x} \cdot \mathbf{n}+\eta|}{\|\mathbf{n}\|}
$$

Obviously, the farthest point from a plane with $\eta=0$ is the closest one to the opposite plane with $\eta=-L_{1}$. In practice, to distinguish the nearest and the farthest points, one can determine the two distances corresponding to the two solutions at fixed $\mathbf{n}$ and $\eta$ and then take the minimum. We denote by $\mathbf{x}_{\min }$ the solution (15) which minimizes the distance $d(\mathcal{E}, \mathcal{P})$. The corresponding algorithm is detailed in A. $\Delta_{2}$ is the minimum distance and $\bar{\Delta}_{2}$ denotes the quantity $\mathbf{n} \cdot \mathbf{x}_{\min }+\eta$.

\subsection{RSA Step 4: Enforce periodicity of the cuboidal cell}

To impose the periodicity of the microstructure in the cuboidal cell, any inclusion $i$ that intersects any of the cuboidal cell faces $j$ defined by the normal $\mathbf{n}$ and $\eta$ is relocated at the opposite face by adding or subtracting the vector $\mathbf{m}$ to the center of the inclusion. To do this, the sign of $\bar{\Delta}_{2}=\mathbf{n} \cdot \mathbf{x}_{\min }+\eta$ must be checked. If the inclusion intersects the face $j, \forall j \in\{1,3,5\}, \bar{\Delta}_{2}<0$, and $\forall j \in\{2,4,6\}$, 
$\bar{\Delta}_{2}>0$. In the more general case that the ellipsoid intersects three orthogonal faces, it is duplicated to the remaining seven corners of the cuboid. If the ellipsoid intersects only two faces then it is duplicated to the three faces that share the same normals. Finally if the ellipsoid intersects only one face, it is duplicated to the opposite face of the cuboid.

\subsection{Illustrative examples}

Figure 3 shows four representative microstructures obtained with the proposed RSA algorithm for a volume fraction $f=20 \%$.

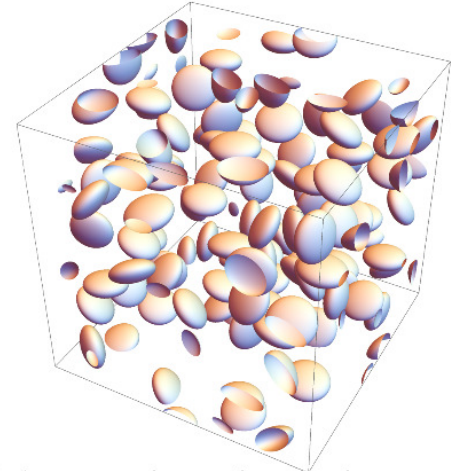

(a)

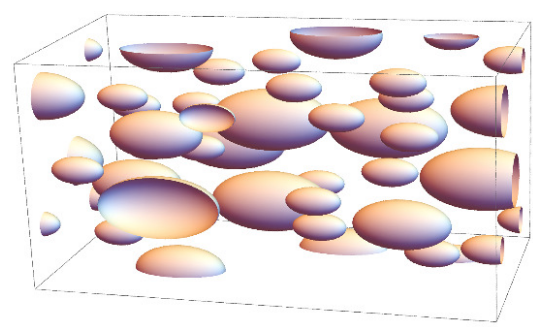

(c)

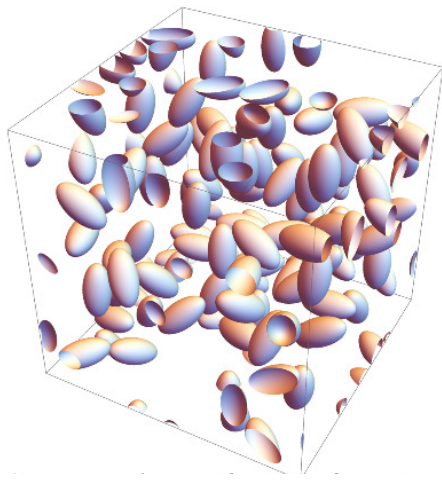

(b)

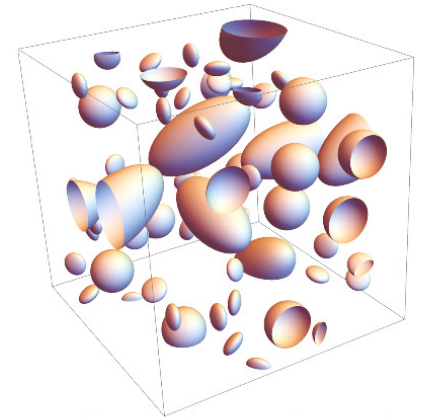

(d)

Figure 3: Illustrative examples of microstructures obtained with the proposed RSA algorithm. Volume fraction $f=20 \%$. (a) and (b): randomly oriented monodisperse in size and shape with $\omega_{1}=\omega_{2}=0.2$ (oblate) and $\omega_{1}=\omega_{2}=5$ (prolate), (c): unidirectional polydisperse in size with $\omega_{1}=\omega_{2}=3$ (prolate) and (d): randomly oriented polydisperse in size and shape.

\section{Determination of effective elastic properties}

In this section, we discuss the computation of the effective elastic properties of periodic porous random particulate materials with an elastic isotropic matrix by considering $3 \mathrm{D}$ cubic unit-cells $V$ 
$\left(L_{1}=L_{2}=L_{3}=L=1\right)$ with periodic boundary conditions. We analyse monodisperse and polydisperse microstructures consisting of random and uniform void distributions with three types of shapes; (i) spherical pores, (ii) prolate spheroidal pores $\left(\omega_{1}=\omega_{2}=\omega>1\right)$ with aspect ratios $\omega=2$ and $\omega=5$ and (iii) oblate spheroidal pores with aspect ratios $\omega=0.1$ and $\omega=0.5$. The effective elastic properties of those microstructures are obtained through numerical periodic homogenization. In all subsequent calculations, the elastic properties of the matrix phase are set to: Young's modulus $E=1 \mathrm{GPa}$ and Poisson's ratio $\nu=0.3$.

\subsection{Numerical homogenization}

The numerical computation of the linear effective elastic properties is carried out using the fast Fourier Transform (FFT) numerical scheme and the finite element (FE) method. The FEM calculations have been performed with the commercial software ABAQUS in the context of linear elasticity and small strains. The application of the periodicity conditions is discussed in the next section. The FFT-based numerical approach has been proposed by Moulinec and Suquet [38] to solve periodic boundary-valued heterogeneous unit-cell problems. The principle of this method is to solve iteratively the implicit integral equation for the strain field $\varepsilon(\mathbf{x})$

$$
\varepsilon(\mathbf{x})=\mathbf{E}+\int_{V} \Gamma^{(0)}\left(\mathbf{x}-\mathbf{x}^{\prime}\right): \boldsymbol{\tau}\left(\mathbf{x}^{\prime}\right) \mathrm{d} \mathbf{x}^{\prime}, \quad \boldsymbol{\tau}(\mathbf{x})=\left(\mathbb{C}(\mathbf{x})-\mathbb{C}^{(0)}\right): \varepsilon(\mathbf{x})
$$

with $\mathbf{E}$ the macroscopic strain and $\boldsymbol{\Gamma}^{(0)}$ denoting the strain Green operator corresponding to a reference homogeneous medium with elasticity $\mathbb{C}^{(0)}$. Following [39], (17) is usually called the Lippman-Schwinger equation by analogy with the quantum mechanics scattering theory. Developments of the FFT-based method have been proposed by several authors, in particular to improve its convergence in the case of a high mechanical contrast on the local properties [see, among others, 40, 41, 42, 43]. In the present study, we have used the augmented Lagrangian scheme originally proposed by Michel et al. [41] and later reinterpreted by Moulinec and Silva [44] as a special case of the polarization-based scheme of Monchiet and Bonnet [43].

At this point it is important to note that the FE approach may require tedious meshing that more than often does not converge, especially for penny-shaped (resp. elongated) inclusions presenting very low (resp. high) aspect ratios. This is the case in the present work for oblate spheroids with aspect ratio lower than 0.3 and for prolate spheroids with aspect ratio higher than 3. By contrast, in the FFT method, a regular grid is employed to discretize the unit-cell (i.e. voxel-based meshing). To describe accurately the highly elongated ellipsoids, a large number of voxels is required but finally yields to converged results. A convergence analysis with respect to the number of voxels, the number of pores and the number of realizations is presented in B. To assess the relative accuracy of the results obtained 
with the FFT-based method and the FE method, we carry out in Section 3.2 calculations for randomly oriented, monodisperse pore microstructures with aspect ratios equal to $0.5,1$ and 2 .

\subsubsection{Periodic Boundary conditions and effective stiffness tensor}

For both numerical techniques, the unit-cell is subjected to periodic boundary conditions [45, 46]. The displacement field $\mathbf{u}$ can be split into an affine part $\mathbf{E} \cdot \mathbf{x}$ and a periodic correction term $\mathbf{u}^{*}(\mathbf{x})$ such that

$$
\mathbf{u}(\mathbf{x})=\mathbf{E} \cdot \mathbf{x}+\mathbf{u}^{*}(\mathbf{x}), \quad \forall \mathbf{x} \in V
$$

where $\mathbf{E}$ is the overall strain which is equal to the average strain in the unit-cell $(\mathbf{E}=\langle\varepsilon\rangle)^{2}$ and $\mathbf{u}^{*}$ is a periodic field characterizing the fluctuation of the displacement due to the presence of the heterogeneities. The local strain field $\varepsilon(\mathbf{x})$ deriving from the displacement $\mathbf{u}$ thus admits the following decomposition $\varepsilon(\mathbf{x})=\mathbf{E}+\varepsilon^{*}(\mathbf{x})$ with $\left\langle\varepsilon^{*}(\mathbf{x})\right\rangle=\left\langle\nabla_{s} \mathbf{u}^{*}(\mathbf{x})\right\rangle=0$. The components of $\mathbf{u}^{*}$ take identical values at points on opposite faces of the unit-cell, in such a way that, considering a Cartesian frame of reference with origin placed at a corner of the cubic unit cell and axes $\mathbf{e}_{k}(k=1,2,3)$ are aligned with the principal axes of the cubic unit cell, we have [47]

$$
\begin{aligned}
& u_{k}\left(L, x_{2}, x_{3}\right)-u_{k}\left(0, x_{2}, x_{3}\right)=E_{k 1} L, \\
& u_{k}\left(x_{1}, L, x_{3}\right)-u_{k}\left(x_{1}, 0, x_{3}\right)=E_{k 2} L, \\
& u_{k}\left(x_{1}, x_{2}, L\right)-u_{k}\left(x_{1}, x_{2}, 0\right)=E_{k 3} L
\end{aligned}
$$

with $k=(1,2,3)$. These relations constitute simple linear constraints that can be entered in any numerical code with a number of available techniques, such as elimination, Lagrange multipliers and penalty methods. In the context of our FE calculations in the Abaqus software, the linear constraint equations (19) are implemented by use of the $*$ Equation command, which uses the elimination technique. As a practical remark for the finite element calculations, keeping the origin of the unit-cell fixed, loadings were done at its three corners having coordinates $(L, 0,0),(0, L, 0)$ and $(0,0, L)$ since the periodic boundary conditions (19) can be rewritten in terms of the displacements of these three corner nodes (see details in Appendix B of [48]). The equations relating the displacements of the nodes on opposite faces of the unit-cell were coupled to those of the corner nodes. Note that the above procedure can be applied to any cuboidal shape. In the special case of voids, one should make sure that no void (nodeless point) is lying in any of the above four corners.

Once the local stress and strain fields are calculated, the effective stiffness tensor $\widetilde{\mathbb{C}}$ is obtained with

\footnotetext{
${ }^{2}$ In the sequel, the angular brackets $\langle\bullet\rangle$ denote the average of a field over the unit-cell.
} 
the relation between average stress and strain tensors

$$
\langle\boldsymbol{\sigma}\rangle=\widetilde{\mathbb{C}}:\langle\varepsilon\rangle
$$

The computation of the twenty-one independent coefficients of $\widetilde{\mathbb{C}}$ is classically done by considering six

\subsubsection{Deviation from isotropy}

Theoretically, a completely random distribution and orientation of ellipsoidal inclusions leads to an isotropic effective response. In practice, due to the finite number of inclusions and/or pores in the unit-cell, the resulting numerical effective stiffness tensor $\widetilde{\mathbb{C}}$ is not exactly isotropic. To evaluate the deviation from isotropy of the tensor $\widetilde{\mathbb{C}}$, use is made of its isotropic projection $\widetilde{\mathbb{C}}^{\text {iso }}$ on the fourth-order deviatoric and hydrostatic isotropic tensors

$$
\widetilde{\mathbb{C}}^{i s o}=3 \widetilde{\kappa} \mathbb{J}+2 \widetilde{\mu} \mathbb{K} \quad \text { with } \quad \widetilde{\kappa}=\frac{1}{3} \widetilde{\mathbb{C}}:: \mathbb{J}=\frac{1}{9} \widetilde{C}_{i i j j} \quad \text { and } \quad \widetilde{\mu}=\frac{1}{10} \widetilde{\mathbb{C}}:: \mathbb{K}=\frac{1}{10}\left(\widetilde{C}_{i j i j}-9 \widetilde{\kappa}\right) .
$$

Here, $\widetilde{\kappa}$ and $\widetilde{\mu}$ are the isotropic bulk and shear moduli. The isotropic projectors are defined by

$$
\mathbb{J}=\frac{1}{3} \mathbf{i} \otimes \mathbf{i} \quad \text { and } \quad \mathbb{K}=\mathbb{I}-\mathbb{J}
$$

with $\mathbf{i}$ and $\mathbb{I}$ the identity tensors, respectively for symmetric second and fourth-order tensors. Note that $\mathbb{J}$ and $\mathbb{K}$ are orthogonal idempotent tensors, i.e $\mathbb{J}: \mathbb{J}=\mathbb{J}, \mathbb{K}: \mathbb{K}=\mathbb{K}$, and $\mathbb{J}: \mathbb{K}=\mathbb{K}: \mathbb{J}=\mathbb{O}$.

The deviation from isotropy can be quantified in two ways: either geometrically (material symmetry) or mechanically (elasticity symmetry). On the one hand, the geometrical approach ${ }^{3}$ makes use of morphological descriptors such as statistical correlation functions to characterize statistically the microstructure (e.g. two-point probability function is often used to quantitatively ascertain at least lower-order information on the geometrical arrangement of phases). On the other hand, the mechanical approach consists in evaluating the deviation from elastic isotropy by choosing an appropriate measure of the elasticity tensor. One of the first measure of this kind is the well-known Zener ratio [50], for cubic symmetry materials, which is simply the ratio of the shear moduli. Later on, several authors have adressed the more general question of the distance between two elasticity tensors of given symmetry [see, for instance, $51,52,53,54]$. At this point, it is worth recalling that the symmetry of a physical property (elasticity, for instance) is necessarily equal or higher than the material symmetry. Consequently, the

\footnotetext{
${ }^{3}$ This approach has been used by many authors. For instance, Kanit et al. [49] quantitatively characterized the geometry of 3D Voronoï tesselation based on the covariance function related to the covariograms of plane section images of the microstructure while Segurado and Llorca [24] have used the radial distribution function of sphere centroids to assess the randomness of the particle distribution, and hence the isotropy of the microsctructure.
} 
geometrical isotropy implies the mechanical isotropy but not the other way around. In addition, in the RVEs studied here one can never obtain an exact geometrical isotropy due to the finite number of the embedded inclusions and the periodicity of the unit cell. In this case then, it is extremely difficult to estimate quantitatively the deviation from mechanical isotropy (which is the measure of interest in this study) by using the deviation from geometrical isotropy.

The present study is, thus, focused on the analysis of the isotropy of the overall elastic response for different random distributions of ellipsoidal voids in the matrix. The deviation from isotropy $\delta_{i s o}$ of the effective elasticity tensor $\widetilde{\mathbb{C}}$ is evaluated with the normalized common Euclidean distance

$$
\delta_{i s o}=\frac{\left\|\widetilde{\mathbb{C}}-\widetilde{\mathbb{C}}^{i s o}\right\|_{F}}{\|\widetilde{\mathbb{C}}\|_{F}}
$$

where $\|\mathbb{A}\|_{F}=\sqrt{\operatorname{tr}\left(\mathbb{A}: \mathbb{A}^{\mathrm{T}}\right)}$ is the Frobenius norm of the tensor $\mathbb{A}$. The value of $\delta_{i s o}=0$ thus corresponds to isotropy. This approach has been previously used, for example, in [55] for the case of linear elastic response of ellipsoidal particles reinforced composites, as well as for nonlinear mechanical responses [27, 20].

\subsection{Assessment of FFT and FE numerical computations}

FE calculations of the effective elastic properties were carried out using the FE package [56], only on monodisperse microstructures with pores aspect ratios $0.5,1$ and 2 and volume fractions $5 \%$ and $15 \%$. The mesh generator NETGEN [57] was used to create meshes of the unit-cell with ten-node quadratic tetrahedral elements (C3D10 in ABAQUS notation). For certain microstructures containing spheroidal pores, we faced severe convergence issues with the meshing procedure when using quadratic ten-node elements. To overcome these difficulties, we have resorted to linear four-node tetrahedral elements (C3D4 in ABAQUS notation) and substantial increase of the number of elements to compensate for the inaccuracy of the linear shape functions. For microstructures with spherical voids, a number of pores $N=30$ is found to be sufficient for convergence. However, for non-spherical voids and large porosities, convergence could require a much larger number of pores (more than $N=500$ ). Unfortunately, in that case the FEM calculations become substantially heavy. To keep the number and time of FEM calculations tractable, we have chosen to use $N=100$ for microstructures with spheroidal pores to get only an idea of the FEM outcome. This issue has motivated the use of the FFT numerical scheme in the rest of this study.

The FFT calculations have been performed on a regular grid with $128 \times 128 \times 128$ voxels. The choice of this spatial resolution is justified by the convergence analysis in terms of number of voxels presented in B (Figure 1). In particular, for monodisperse prolate and oblate spheroids with aspect ratios $\omega_{1}=\omega_{2}=2$ and 0.5 , respectively, a number of pores $N=500$ was found to be sufficient for 
achieving representativity (Figure 2). In the case of spherical voids, $N=30$ pores are sufficient to obtain accurate results as for the FE computations. Note that the material properties have been spheroidal prolate and oblate void (i.e. $\omega_{1}=\omega_{2}=\omega$ ). In the sequel, $\omega=1$ refers to spherical, $\omega<1$ for oblate and $\omega>1$ for prolate voids. 
Next, it is recalled that $N^{\text {ref }}=N=500$ for the case of monodisperse microstructures. For deviation between various realizations both in mono- and polydisperse microstructures is extremely small and for that reason no error bars are shown in the results that follow.

The numerical FFT results are also compared with HSW analytical estimates which are obtained by employing the approach proposed by Gatt et al. [15], see also [59], for isotropic composites comprising randomly oriented polydisperse ellipsoidal voids. In Figure 4 , the deviation from isotropy $\delta_{i s o}(c . f$.

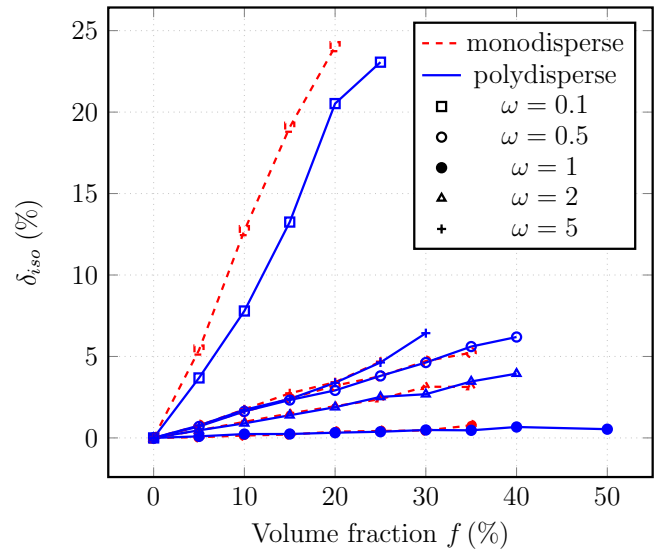

Figure 4: Deviation from isotropy in monodisperse microstructures compared against that in polydisperse microstructures for different pores aspect ratios $\omega=0.1,0.5,1,2$ and 5 .

equation (23)) is shown for monodisperse and polydisperse microstructures. Hence, the spherical voids lead to the most isotropic effective response with $\delta_{i s o}<0.5 \%$ for both monodisperse and polydisperse distributions. As one increases or decreases the aspect ratios from unity to get either prolate or oblate shapes, the deviation from isotropy increases. Even for a high number of inclusions, the transversely isotropic shape of the void affects the evaluation of the elastic properties. The deviation is markedly strong for oblate voids with aspect ratios $\omega=0.1$, where $\delta_{i s o} \sim 24 \%$ for both monodisperse and 
polydisperse distributions at volume fractions $f>15 \%$. This implies that even if theoretically one can consider very large number of orientations, in practice, with our present RSA method, a significant effect of the void shapes remains and lead to an anisotropic response.

In the rest of the study, we show only the isotropic parts corresponding to spheroidal voids and compare them with exactly isotropic (i.e. infinite number of orientations) analytical results. Nonetheless, their interpretation should be done with caution since the numerical estimates exhibit in some cases (such as $\omega=0.1$ ) strong deviation from isotropy. Moreover, it is noted that using a larger number of realizations does not reduce significantly the deviation from isotropy in these extreme cases since

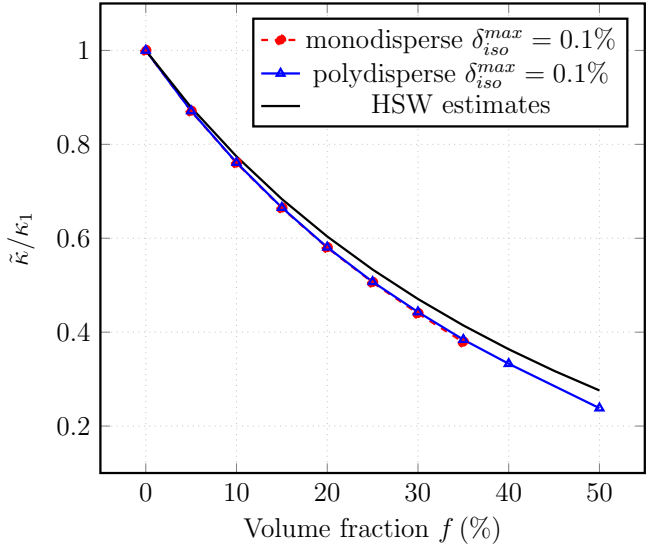

(a)

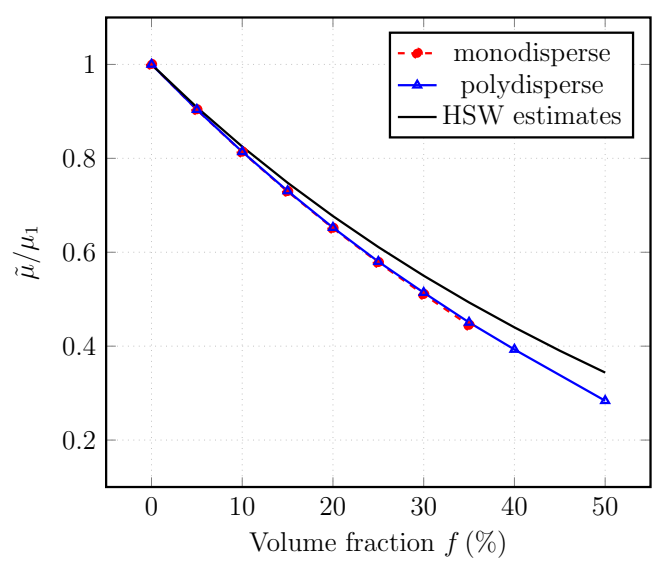

(b)

Figure 5: Comparison between FFT and HSW estimates for monodisperse and polydisperse microstructures with spherical pores. Effective moduli as a function of porosity: (a) normalized bulk modulus $\widetilde{\kappa} / \kappa_{1}$ and (b) shear modulus $\widetilde{\mu} / \mu_{1}$

In Figure 6, FFT and HSW estimates for prolate monodisperse and polydisperse voids with aspect ratios $\omega=2$ and 5 are reported. First, it should be noted that by referring to Figure 4 , the maximum deviation from isotropy occurs for $\omega=5$ and is $\delta_{i s o}=6 \%$. Therefore, the curves shown correspond to 
only the isotropic part of the elastic properties as defined in (21). Again a minor difference is observed between the mono- and polydisperse microstructures with the later being only slightly more compliant. shear modulus $\widetilde{\mu}$.

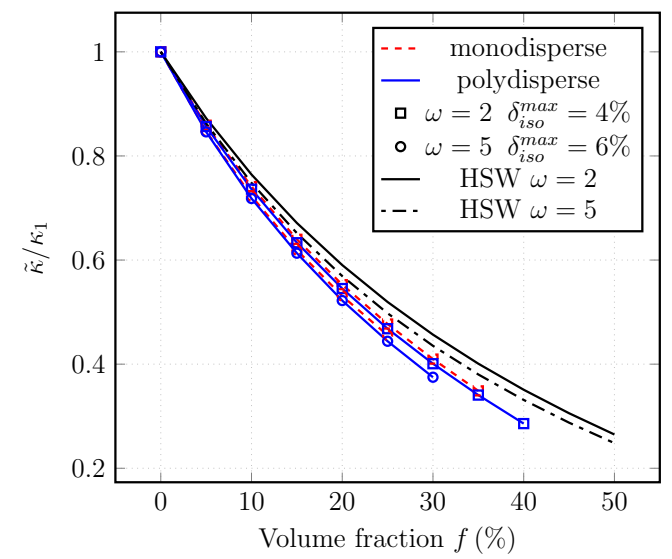

(a)

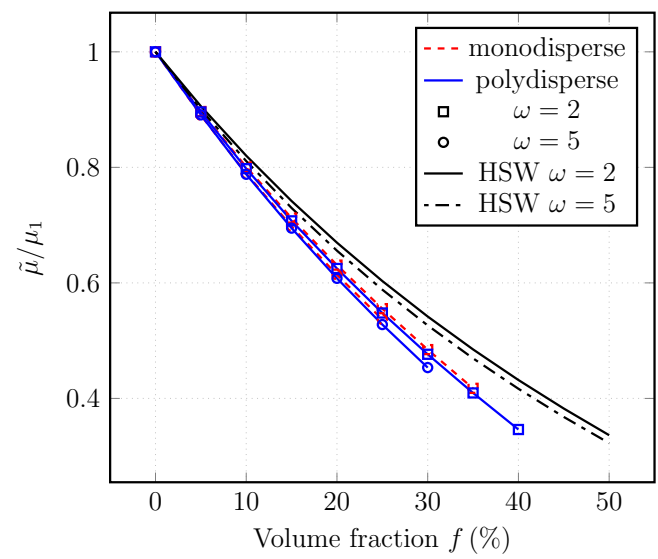

(b)

Figure 6: Comparison between FFT and HSW estimates for monodisperse and polydisperse microstructures with prolate spheroidal pores. Effective moduli as a function of porosity: (a) normalized bulk modulus $\widetilde{\kappa} / \kappa_{1}$ and (b) shear modulus $\widetilde{\mu} / \mu_{1}$

Similar observations can be done also in the context of oblate voids in Fig. 7. The estimates corresponding to the very elongated voids with $\omega=0.1$ are substantially more compliant than the rest. It is interesting to observe that the HSW analytical estimates are very close to the numerical estimates but it is recalled that for $\omega=0.1$ the deviation from isotropy is very large $\delta_{i s o}=24 \%$. Moreover, one can observe that the effect of the aspect ratio is more pronounced on the bulk modulus $\widetilde{\kappa}$ than in the shear modulus $\widetilde{\mu}$. We also note that in such extreme cases of elongated ellipsoids, it is progressively more difficult to reach higher volume fractions even with polydisperse sizes. For instance, in the case of $\omega=0.1$, we were able to reach $f=20 \%$ for the monodisperse and $f=25 \%$ for the polydisperse.

\subsection{Effect of aspect ratio in polydisperse microstructures}

Figure 8 shows a comprehensive plot of the effect of the aspect ratio for polydisperse microstructures upon the effective bulk and shear moduli. We observe that spheroidal (prolate and oblate) voids tend to make the material more compliant as already seen in a number of studies [see, for instance, 15]. The oblate voids tend to lead to stronger drops of both $\widetilde{\kappa}$ and $\widetilde{\mu}$ than prolate ones but one again should recall the strong deviation from isotropy for the oblate voids. We close by noting that the spherical voids lead to the less compliant response and hence are better candidates for materials that can approach the Hashin-Shtrikman bounds. 


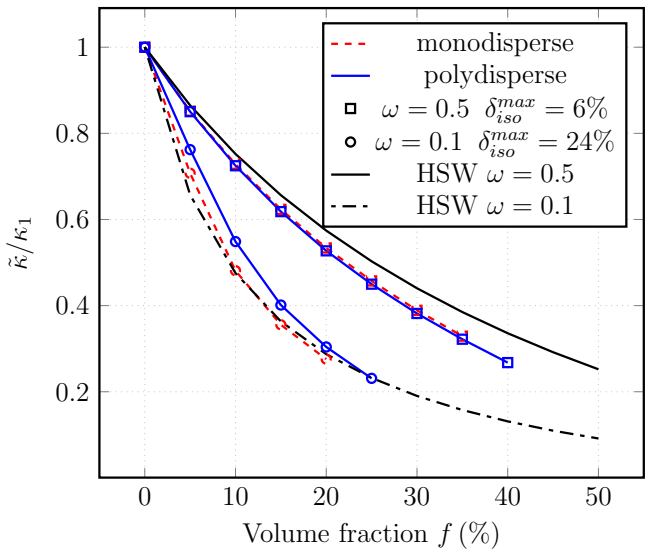

(a)

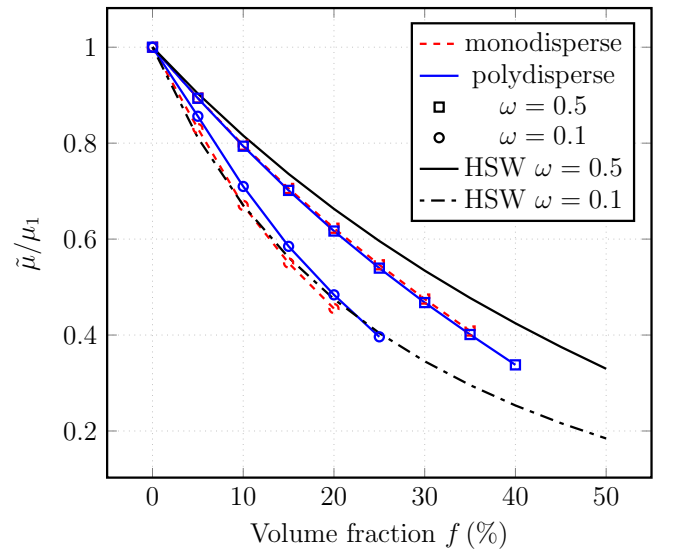

(b)

Figure 7: Comparison between FFT and HSW estimates for monodisperse and polydisperse microstructures with oblate spheroidal pores. Effective moduli as a function of porosity: (a) normalized bulk modulus $\widetilde{\kappa} / \kappa_{1}$ and (b) shear modulus $\widetilde{\mu} / \mu_{1}$.

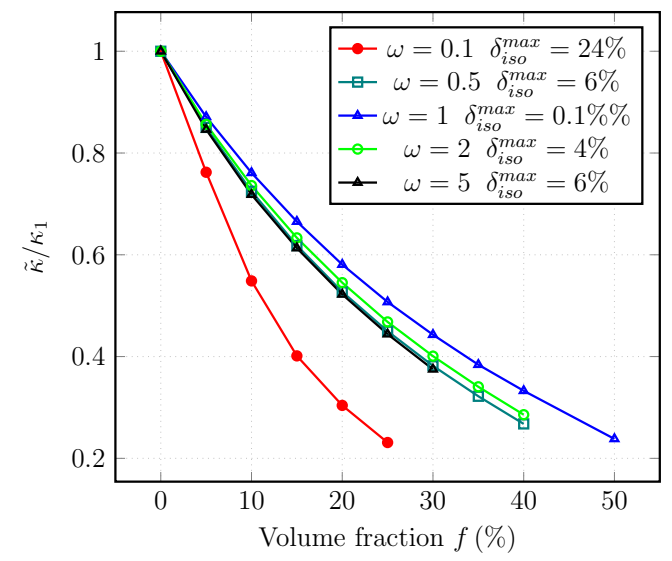

(a)

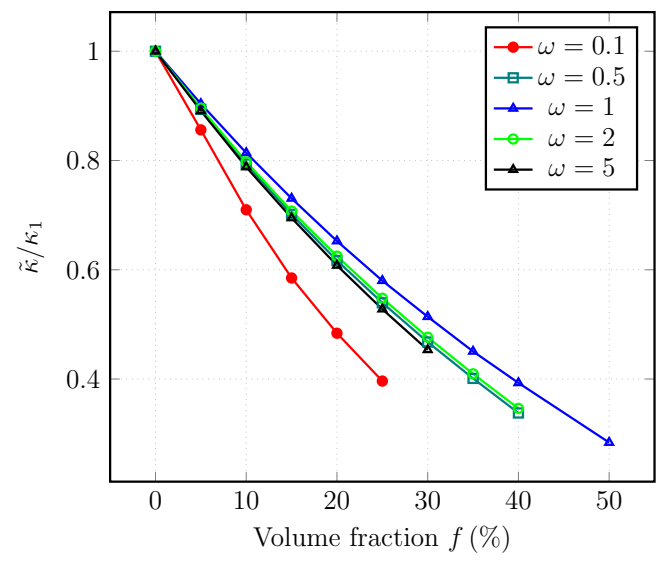

(b)

Figure 8: Influence of the pore aspect ratio $\omega$ on the evolution of the effective moduli as a function of the porosity: (a) normalized bulk modulus $\widetilde{\kappa} / \kappa_{1}$ and (b) shear modulus $\widetilde{\mu} / \mu_{1}$.

\section{Concluding Remarks}

In the present study, we extend the classical Random Sequential Adsorption (RSA) algorithm to

425

the context of randomly oriented ellipsoidal inclusions of arbitrary size, shape and orientation (i.e. polydisperse). The main algorithm is based upon a proper simplification of Lin and Han [36] algorithm, which consists in employing a more general way to check the non-overlapping condition of inclusions during the sequential addition process. In that algorithm, the non-overlapping condition is checked by solving two one-dimensional quadratic inequalities at each iteration. Instead, with our modification, we are able to check this condition once by using a lemma on the intersection of ellipsoids proposed in the 
book of Kurzhanski and Vályi [37]. The proposed algorithm is described in detail, is robust and can be easily implemented.

Secondly, the present study analyzes numerically the effective elastic properties of a large number of representative volume elements (RVEs) including monodisperse as well as polydisperse voids randomly distributed in the unit cell. Those numerical estimates are probed by the classical Hashin-ShtrikmanWillis (HSW) bounds and estimates. The deviation from isotropy of the numerically-obtained effective elastic tensors is critically assessed by using a simple measure based on a normalized Euclidean distance between two fourth order tensors and the Frobenius norm. In simple words, we project the numerically obtained effective elastic tensor (which may exhibit certain degree of anisotropy) to the isotropic fourth order tensor space to obtain an "isotropized" one. The Euclidean difference of the original tensor and the isotropized one, gives a simple to understand scalar measure of the deviation of isotropy.

In this regard, we find that as the aspect ratios of the voids increase (or decrease) substantially, the proposed RSA algorithm leads to substantially anisotropic porous materials which can deviate from isotropy by as much as $24 \%$ for oblate voids with aspect ratio $\omega_{1}=\omega_{2}=0.1$. This deviation is present even for very large number of voids $N>500$ both in the case of monodisperse and polydisperse microstructures. The polydisperse microstructures exhibit in principle lower anisotropy albeit an important one for $\omega_{1}=\omega_{2}=0.1$ (reaching 20\%). Interestingly, the isotropized shear and bulk moduli corresponding to the very elongated void aspect ratios remain in good agreement with the corresponding isotropic HSW estimates. As expected by previous works, the spherical voids exhibit the stiffest response out of all cases considered.

In closing, we remark that the present RSA microstructures can be used as a test bed for nonlinear analytical homogenization models in the context of finite strains and nonlinear mechanical and multiphysics responses (see for instance studies in nonlinear elasticity [27], in elasto-plasticity [47, 62], as well as in nonlinear magneto-elasticity $[48,63])$. Furthermore, with the current 3D printing technology such RVEs can be readily printed and experimentally tested in the lab. Such work is in progress [61]. Finally, the present algorithm can be potentially used to generate, 3D print and analyze microstructures where the aspect ratios of the inclusions are provided by external sources (for instance imaging or 3D tomography) via the use of sufficiently defined probability density functions (PDFs) (see for instance the very recent work of [64] in this context).

\section{Acknowledgments}

K.D. would like to thank Prof. P. Suquet for useful comments on improving further the isotropy of the RVEs with very elongated inclusions, which will be included in a future work. The authors wish to acknowledge support from TOTAL S.A. K.D. also acknowledges support from the European Research 
Council (ERC) under the European Unions Horizon 2020 research and innovation program (Grant agreement no. 636903-MAGNETO).

\section{A. Extended RSA algorithm for randomly oriented mono- and polydisperse inclusions}

\section{Nomenclature:}

- $L_{1}, L_{2}, L_{3}$ : dimensions of the cuboidal unit cell

- $f$ : total volume fraction of inclusions

- $N^{\text {ref }}$ : reference number of inclusions in the unit-cell (equal to number of inclusions when monodisperse)

- $R^{r e f}$ : Reference radius of a spherical inclusion

- $N_{p}$ : number of phases/families of inclusions (different shapes or sizes correspond to different families/phases)

- $\omega_{1}^{(r)}, \omega_{2}^{(r)}$ : aspect ratios of the inclusions belonging to family $r$

- $\chi^{(r)}$ : size coefficient defining the allocation of sizes to the different families

- $f^{(r)}$ : volume fraction of each family $r$

- $\xi_{1}, \xi_{2}$ : coefficients controlling the minimum distance between inclusions and between the unit-cell faces and the inclusions

- $\mathbf{v}_{i}^{(r)}(i=1,2,3)$ : position vector of the center of an inclusion belonging to family $r$

- $a_{i}^{(r)}, b_{i}^{(r)}, c_{i}^{(r)}$ : lengths of semi-axes of an inclusion belonging to family $r$

- $\phi_{i}^{(r)}, \theta_{i}^{(r)}, \psi_{i}^{(r)}$ : the three Euler angles defining the orientation of the elliptical inclusion belonging to family $r$

- $\mathbf{m}=\left(m_{1}, m_{2}, m_{3}\right):$ total of 27 vectors defining the translational periodicity of the cuboidal unit cell, where $m_{1}, m_{2}$ and $m_{3}$ take the values in all combinations $0,-L_{1}, L_{1}, 0,-L_{2}, L_{2}$ and $0,-L_{3}, L_{3}$.

- $\Delta_{1}, \Delta_{2}, \bar{\Delta}_{2}$ : Distances between inclusion-inclusion and inclusion-cuboidal face

- $\mathbb{Z}^{(r)}$ : matrix describing the shape and orientation of inclusion belonging to family $r$ 


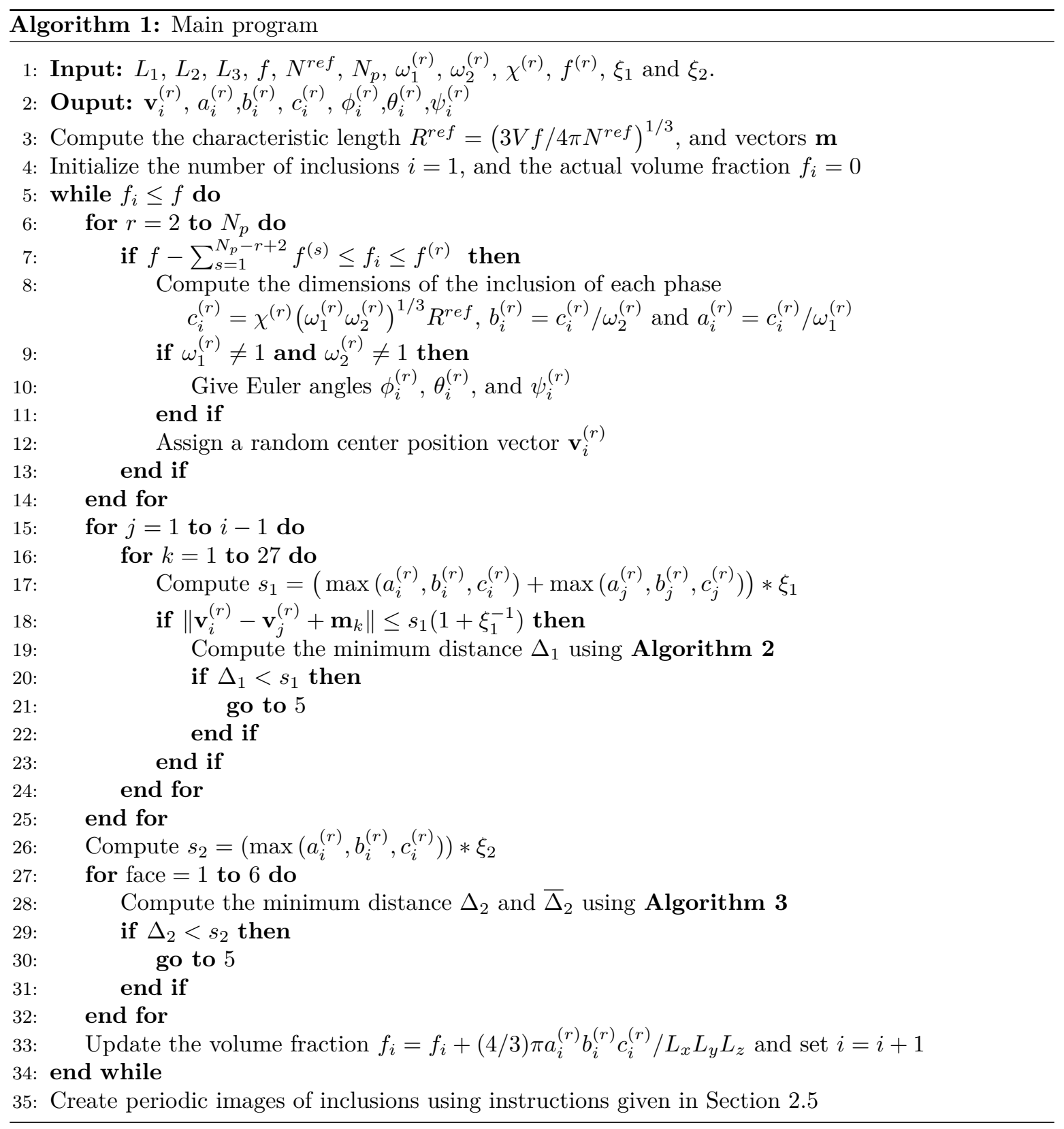



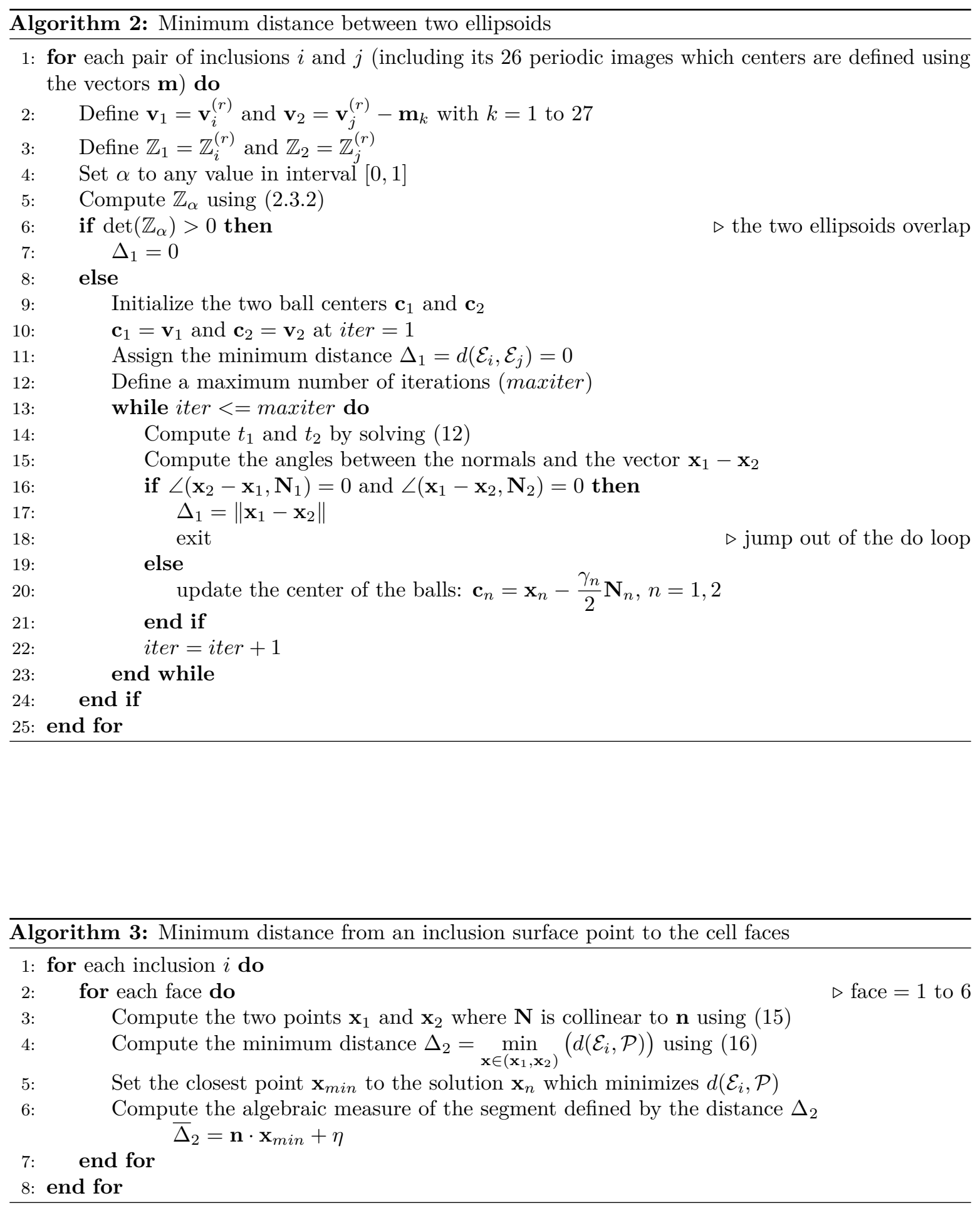


\section{B. Convergence analysis}

\section{B.1. Convergence of FFT spatial resolution}

The convergence analysis in terms of the number of voxels is conducted for three different microstructures made up of monodisperse pores with volume fraction $20 \%$ at a fixed realization. Cubic unit cells were generated comprising a number of pores $N=50$ for microstructure with spherical pores, and $N=500$ for microstructures with oblate $(\omega=0.5)$ and prolate $(\omega=2)$ spheroidal pores.

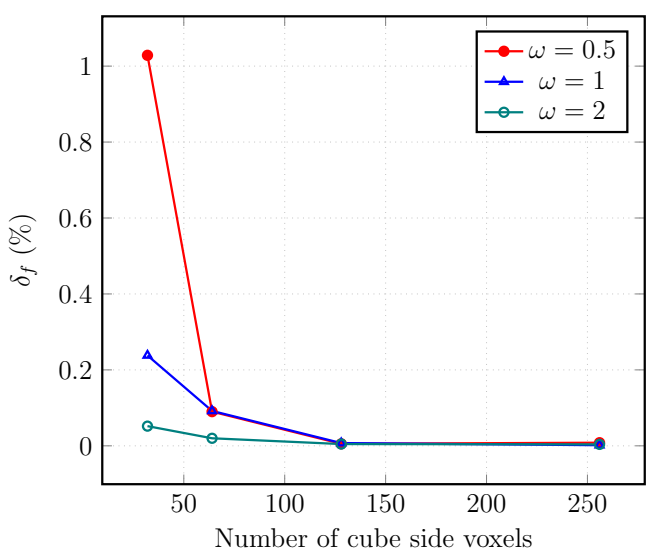

(a)

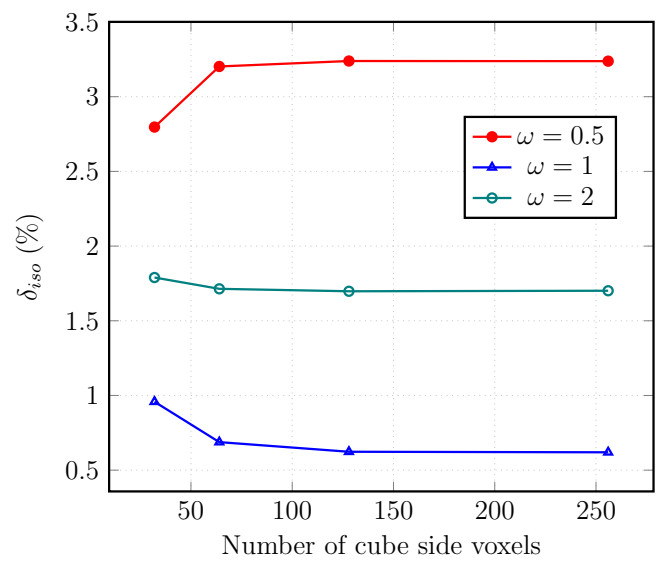

(b)

Figure 1: Convergence analysis in terms of the number of voxels performed on three different microstructures made up of monodisperse with volume fraction $20 \%$ at fixed realization: (a) error on the volume fraction $\delta_{f}$ and (b) deviation from isotropy $\delta_{i s o}$ as a function of the number of voxels along each side of the unit-cell (32, 64, 128 and 256).

Specifically, each microstructure was discretized with grid of $32^{3}, 64^{3}, 128^{3}$ and $256^{3}$ voxels. The error committed on the real volume fraction, denoted by $\delta_{f}$, was evaluated for each discretization (Figure 1(a))

$$
\delta_{f}=\frac{\left|f-f_{\text {voxel }}\right|}{f}
$$

\section{B.2. Convergence in terms of number of inclusions/pores}

In this section, a convergence analysis in terms of the number of pores was performed on microstructures with prolate $(\omega=2)$ and oblate $(\omega=0.5)$ spheroidal pores with a volume fraction of $20 \%$. At 
fixed realization, cubic unit cells containing monodisperse randomly distributed and oriented spheroidal pores were generated. Each microstructure was discretized with $128^{3}$ voxels, as discussed in the previous section.

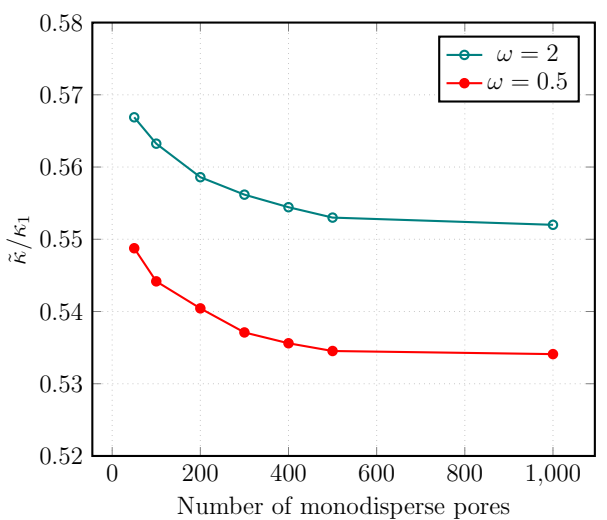

(a)

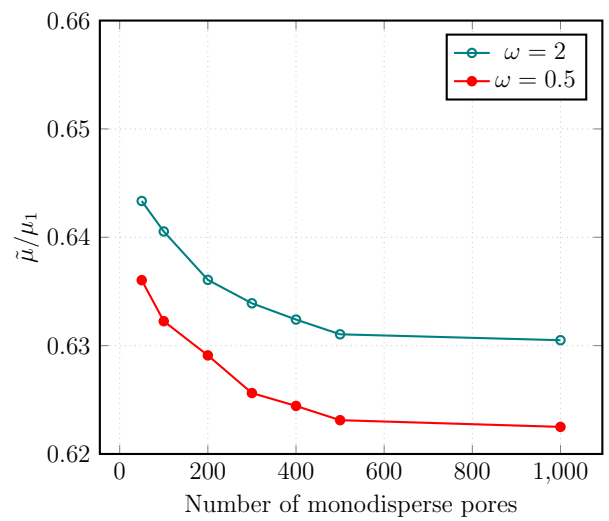

(b)

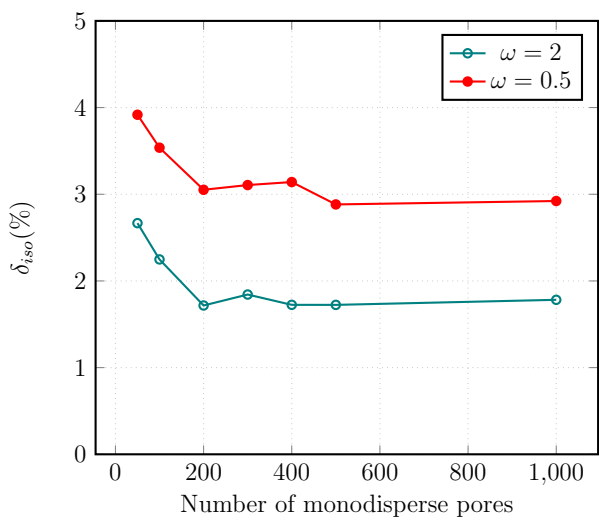

(c)

Figure 2: Convergence in terms of the number of spheroidal pores with aspect ratios $\omega=2$ and $\omega=0.5$ conducted on monodisperse microstructures. Effective moduli and the deviation from isotropy as a function of the number of pores $\left(50,100,200,300,400,500\right.$ and 1000): (a) normalized bulk modulus $\widetilde{\kappa} / \kappa_{1}$, (b) shear modulus $\tilde{\mu} / \mu_{1}$ and (c) isotropy measure.

We can observe in Figure 2(a,b) that for a number of spheroidal pores $N=500$, the normalized elastic moduli $\widetilde{\kappa}$ and $\widetilde{\mu}$ do not change significantly. In parallel, a convergence is also obtained on the result for $\delta_{i s o}$ (Figure $2(c)$ ). Nonetheless, this converged value does not vanish and takes values which can be rather high for oblate voids with very low aspect ratio (Figure 4). This implies that further increase of very elongated inclusions might not necessarily lead to a fully isotropic response. This point deserves further investigation. 


\section{B.3. Convergence in terms of realizations}

Convergence of the effective moduli is studied in terms of realizations at fixed number of pores, volume fraction and discretization. Monodisperse microstructures containing 50 spherical pores $(\omega=1)$ and 500 spheroidal pores with aspects ratios $\omega=0.5,2,5$ were investigated at volume fraction $20 \%$ and discretized with $128^{3}$ voxels.

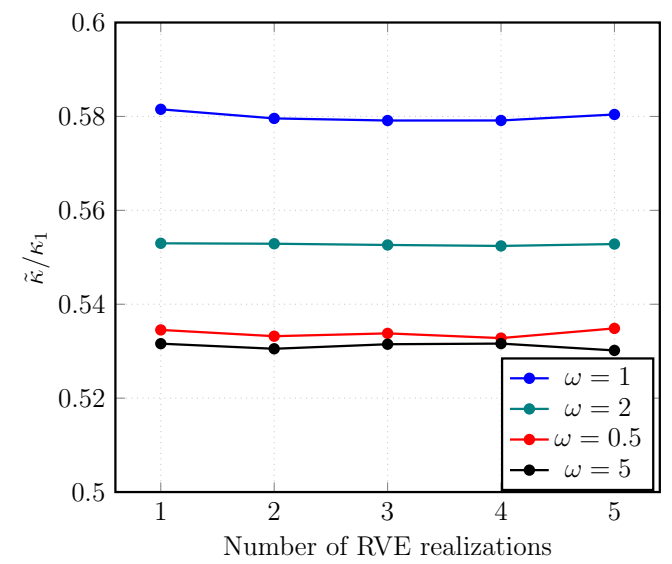

(a)

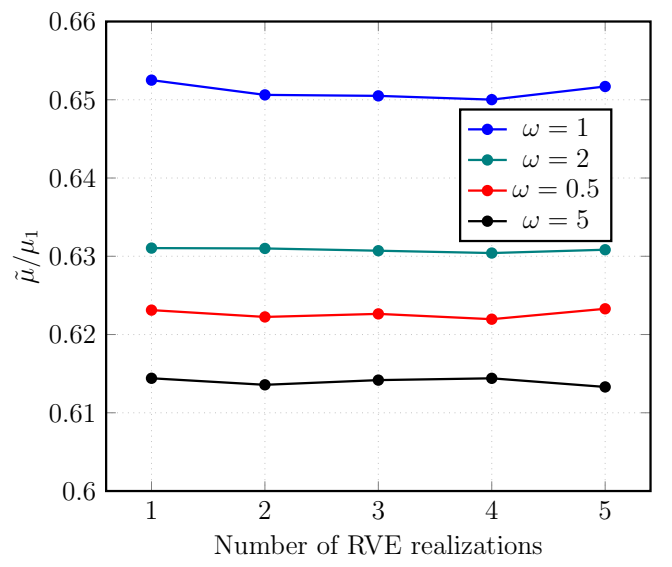

(b)

Figure 3: Effective moduli for five different realizations of monodisperse microstructures with 50 spherical pores $(\omega=1)$ and 500 spheroidal pores with aspects ratios $\omega=0.5,2,5$ and porosity $f=20 \%$ : (a) normalized bulk modulus $\widetilde{\kappa} / \kappa_{1}$ and (b) shear modulus $\tilde{\mu} / \mu_{1}$.

On Figure 3, it can be observed that the normalized effective moduli do not significantly change for different realizations. All reported results in the main text have been averaged over five realizations.

\section{References}

[1] G. W. Milton. The Theory of Composites. Cambridge University Press, 2002.

[2] S. Torquato. Random Heterogeneous Materials: Microstructure and Macroscopic Properties. Springer, New York, 2002.

[3] A. Gillman, G. Amadio, K. Matouš, and T. L." Jackson. Third-order thermo-mechanical properties for packs of platonic solids using statistical micromechanics. Proceedings of the Royal Society of London A: Mathematical, Physical and Engineering Sciences, 471(2177), 2015.

[4] S. Torquato. Random heterogeneous media: Microstructure and improved bounds on effective properties. Appl. Mech. Rev., 44(2):37-76, 1991.

[5] B. Lu and S. Torquato. Linear-path function for random heterogeneous materials. Physical review A, 45(2):922-929, 1992. 
[6] M. J. Beran. Statistical Continuum Theories. Interscience, New York, 1968.

[7] J. R. Willis. Variational and related methods for overall properties of composites. Advances in Applied Mechanics, 21:1-78, 1981.

[8] E. Kröner. Statistical Continuum Mechanics. Springer-Verlag, 1972.

[9] A. K. Sen and S. Torquato. Effective conductivity of anisotropic two-phase composite media. Physical Review B, 39(7):4504-4515, 1989.

[10] A. Mikdam, A. Makradi, S. Ahzi, H. Garmestani, D. S. Li, and Y. Remond. Effective conductivity in isotropic heterogeneous media using a strong-contrast statistical continuum theory. Journal of the Mechanics and Physics of Solids, 57:76-86, 2009.

[11] J. R. Willis. Bounds and self-consistent estimates for the overall modui of anisotropic composites. Journal of the Mechanics and Physics of Solids, 25:185-202, 1977.

[12] P. Ponte Castañeda and J.R. Willis. The effect of spatial distribution on the effective behavior of composite materials and cracked media. Journal of the Mechanics and Physics of Solids, 43(12): 1919 - 1951, 1995. ISSN 0022-5096. doi: https://doi.org/10.1016/0022-5096(95)00058-Q. URL http://www.sciencedirect.com/science/article/pii/002250969500058Q.

[13] Tai Te Wu. The effect of inclusion shape on the elastic moduli of a twophase material. International Journal of Solids and Structures, 2(1):1 - 8, 1966. ISSN 0020-7683. doi: https://doi.org/10.1016/0020-7683(66)90002-3. URL http://www.sciencedirect.com/science/article/pii/0020768366900023.

[14] James G. Berryman. Theory of elastic properties of composite materials. Applied Physics Letters, 35(11):856-858, 1979. doi: 10.1063/1.90982. URL https://doi.org/10.1063/1.90982.

[15] J.-M. Gatt, Y. Monerie, D. Laux, and D. Baron. Elastic behavior of porous ceramics: application to nuclear fuel materials. Journal of Nuclear Materials, 336(2):145 155, 2005. ISSN 0022-3115. doi: https://doi.org/10.1016/j.jnucmat.2004.09.009. URL http://www.sciencedirect.com/science/article/pii/S0022311504006920.

[16] B. D. Lubachevsky and F. H. Stillinger. Geometric properties of random disk packings. Journal of Statistical Physics, 60:561 - 583, 1990.

[17] B.D. Lubachevsky, F.N. Stillinger, and E.N. Pinson. Disks vs. spheres : contrasting properties of random packings. Journal of Statistical Physics, 64:501 - 523, 1991. 
[20] Pierre-Guy Vincent, Pierre Suquet, Yann Monerie, and Hervé Moulinec. Effective flow surface of porous materials with two populations of voids under internal pressure: Ii. full-field simulations. International Journal of Plasticity, 56:74 - 98, 2014.

[21] F. Lavergne, R. Brenner, and K. Sab. Effects of grain size distribution and stress heterogeneity

[27] O. Lopez-Pamies, T. Goudarzi, and K. Danas. The nonlinearelasticresponseofsuspensionsofrigidinclusions in rubber: Ii - a simple explicit approximation for finite-concentration suspensions. Journal of the Mechanics and Physics of Solids, 61:19-37, 2013. 
[28] J D Sherwood. Packing of spheroids in three-dimensional space by random sequential addition. Journal of Physics A: Mathematical and General, 30(24):L839, 1997. URL http: //stacks. iop.org/0305-4470/30/i=24/a=004.

[29] Helmut J. Böhm and Azra Rasool. Effects of particle shape on the thermoelastoplastic behavior of particle reinforced composites. International Journal of Solids and Structures, 87: 90 - 101, 2016. ISSN 0020-7683. doi: https://doi.org/10.1016/j.ijsolstr.2016.02.028. URL http://www.sciencedirect.com/science/article/pii/S0020768316000901.

[30] S. Kari, H. Berger, R. Rodriguez-Ramos, and U. Gabbert. Computational evaluation of effective material properties of composites reinforced by randomly distributed spherical particles. Composite Structures, 77:223 - 231, 2007.

[31] C. Sun, P. Saffari, R. Ranade, K. Sadeghipour, and G. Baran. Finite element analysis of elastic property bounds of a composite with randomly distributed particles. Composites Part A: Applied Science and Manufacturing, 38:80 - 86, 2007.

[32] A. Jean, F. Willot, S. Cantournet, S. Forest, and D. Jeulin. Large-scale computations of effective elastic properties of rubber with carbon black fillers. International Journal for Multiscale Computational Engineering, 9:271-303, 2011.

[33] I. Papadioti, K. Danas, and N. Aravas. A methodology for the estimation of the effective yield function of isotropic composites. International Journal of Solids and Structures, 87: 120 - 138, 2016. ISSN 0020-7683. doi: https://doi.org/10.1016/j.ijsolstr.2016.02.022. URL http://www.sciencedirect.com/science/article/pii/S0020768316000846.

[34] O. Pierard, C. Gonzalez, J. Segurado, J. LLorca, and I. Doghri. Micromechanics of elasto-plastic materials reinforced with ellipsoidal inclusions. International Journal of Solids and Structures, 44: 6945-6962, 2007.

[35] Xiao-Diao Chen, Jun-Hai Yong, Guo-Qin Zheng, Jean-Claude Paul, and Jia-Guang Sun. Computing minimum distance between two implicit algebraic surfaces. Computer-Aided Design, 38:1053 - 106, 2006.

[36] A. Lin and S-P Han. On the distance between two ellipsoids. Society for Industrial and Applied Mathematics, 13(1):298-308, 2002.

[37] Alexander Kurzhanski and István Vályi. Ellipsoidal calculus for estimation and control. 1997. 
[38] H. Moulinec and P. Suquet. A numerical method for computing the overall response of nonlinear composites with complex microstructure. Computer Methods in Applied Mechanics and Engineering,

[39] E. Kröner. Bounds for effective elastic moduli of disordered materials. J. Mech. Phys. Solids, 25: 137-155, 1977.

[40] D. J. Eyre and G. W. Milton. A fast numerical scheme for computing the response of composites using grid refinement*. Eur. Phys. J. AP, 6(1):41-47, 1999.

[41] J.C. Michel, H. Moulinec, and P Suquet. A computational method based on augmented lagrangians and fast fourier transforms for composites with high contrast. Computer Modeling in Engineering and Sciences, 1(2):79-88, 2000.

[42] S. Brisard and L. Dormieux. Fft-based methods for the mechanics of composites: A general variational framework. Computational Materials Science, 49(3):663 - 671, 2010.

[43] V. Monchiet and G. Bonnet. A polarization-based fft iterative scheme for computing the effective properties of elastic composites with arbitrary contrast. International Journal for Numerical Methods in Engineering, 89(11):1419-1436, 2012.

[44] H. Moulinec and F. Silva. Comparison of three accelerated fft-based schemes for computing the mechanical response of composite materials. International Journal for Numerical Methods in Engineering, 97(13):960-985, 2014.

[45] P. Suquet. Homogenization techniques for composite media. In Lecture notes in Physics, pages 194-278. Springer-Verlag, 1987.

[46] J.C. Michel, H. Moulinec, and P. Suquet. Effective properties of composite materials with periodic microstructure: a computational approach. Computer Methods in Applied Mechanics and Engineering, 172(1-4):109 - 143, 1999.

[47] A. Mbiakop, A. Constantinescu, and K. Danas. An analytical model for porous single crystals with ellipsoidal voids. Journal of the Mechanics and Physics of Solids, 84: 436 - 467, 2015. ISSN 0022-5096. doi: https://doi.org/10.1016/j.jmps.2015.07.011. URL http://www. sciencedirect.com/science/article/pii/S002250961530017X.

[48] K. Danas. Effective response of classical, auxetic and chiral magnetoelastic materials by use of a new variational principle. Journal of the Mechanics and Physics of Solids, 105: 25 - 53, 2017. ISSN 0022-5096. doi: https://doi.org/10.1016/j.jmps.2017.04.016. URL http://www.sciencedirect.com/science/article/pii/S0022509616309140. 
[49] T. Kanit, S. Forest, I. Galliet, V. Mounoury, and D. Jeulin. Determination of the size of the representative volume element for random composites: statistical and numerical approach. International Journal of Solids and Structures, 40(13-14):3647 - 3679, 2003.

[50] C. Zener. Elasticity and anelasticity of Metals. University Chicago Press, 1948.

[51] D. C. Gazis, I. Tadjbakhsh, and R. A. Toupin. The elastic tensor of given symmetry nearest to an anisotropic elastic tensor. Acta Crystallographica, 16(9):917-922, Sep 1963. doi: 10.1107/S0365110X63002449. URL https://doi.org/10.1107/S0365110X63002449.

[52] M. François, G. Geymonat, and Y. Berthaud. Determination of the symmetries of an experimentally determined stiffness tensor: Application to acoustic measurements. International Journal of Solids and Structures, 35(31):4091 - 4106, 1998. ISSN 0020-7683. doi: https://doi.org/10.1016/S0020-7683(97)00303-X. URL http://www.sciencedirect.com/science/article/pii/S002076839700303X.

[53] Maher Moakher and Andrew N. Norris. The closest elastic tensor of arbitrary symmetry to an elasticity tensor of lower symmetry. Journal of Elasticity, 85(3):215-263, 2006.

[54] Ioan Bucataru and Michael A. Slawinski. Invariant properties for finding distance in space of elasticity tensors. Journal of Elasticity, 94(2):97-114, 2008.

[55] Elias Ghossein and Martin Lévesque. A comprehensive validation of analytical homogenization models: The case of ellipsoidal particles reinforced composites. Mechanics of Materials, 75:135 $150,2014$.

[56] ABAQUS Version 6.11. Documentation. Dassault Systémes Simulia Corp, Providence, RI, USA, 2011.

[57] J. Schöberl. Netgen an advancing front 2d/3d-mesh generator based on abstract rules. Computing and Visualization in Science, 1(1):41-52, 1997.

[58] Lionel Gélébart and Franck Ouaki. Filtering material properties to improve fft-based methods for numerical homogenization. Journal of Computational Physics, 294:90 95, 2015. ISSN 0021-9991. doi: https://doi.org/10.1016/j.jcp.2015.03.048. URL http://www.sciencedirect.com/science/article/pii/S002199911500203X.

[59] L.J. Walpole. On the overall elastic moduli of composite materials. Journal of the Mechanics and Physics of Solids, 17(4):235 - 251, 1969. 
[60] G. Zhang and S. Torquato. Precise algorithm to generate random sequential addition of hard hyperspheres at saturation. Phys. Rev. E, 88:053312, Nov 2013. doi: 10.1103/PhysRevE.88.053312.

[61] K. Danas O. Zerhouni, M. G. Tarantino. Numerically-aided 3d printed random isotropic porous materials approaching the hashin-shtrikman bounds. submitted, 2018.

[62] L. Cheng, K. Danas, A. Constantinescu, and D. Kondo. A homogenization model for porous ductile solids under cyclic loads comprising a matrix with isotropic and

65] Elias Ghossein and Martin Lévesque. A fully automated numerical tool for a comprehensive validation of homogenization models and its application to spherical particles reinforced composites. International Journal of Solids and Structures, 49(11-12):1387 - 1398, 2012. 